\title{
1 New Technologies can Cost-effectively Reduce Oil and 2 Gas Methane Emissions, but Policies will Require Careful 3 Design to Establish Mitigation Equivalence
}

\section{Chandler Kemp and Arvind P. Ravikumar}

\section{Email: ckemp@harrisburgu.edu, aravikumar@harrisburgu.edu} Twitter: @arvindpawan1

Department of Systems Engineering, Harrisburg University of Science and Technology, Harrisburg PA 17101 
Methane emissions from petroleum and natural gas systems accounted for $28 \%$ of US methane emissions in 2018, based on the Environmental Protection Agency's (EPA) greenhouse gas inventory (GHGI) [1]. Furthermore, several recent studies have shown that official GHGI estimates likely underestimate methane emissions from natural gas systems [2-6]. Methane is the primary constituent of natural gas and has a global warming potential 34 times greater than that of carbon dioxide over 100 years [2]. Therefore, reducing methane emissions from oil and gas operations is critical to realize GHG emissions benefits from recent coal-to-gas fuel switching in the power sector [7]-[9]. In addition, addressing methane emissions reduces volatile organic compounds co-emitted from natural gas production facilities, thereby improving local air quality [10]. Most importantly, minimizing methane leakage is necessary to achieve long-term climate targets. Gas production continues through 2040 in all pathways considered by the Intergovernmental Panel on Climate Change (IPCC) Special Report on global warming of $1.5^{\circ} \mathrm{C}$, and persists at greater than $20 \%$ of the 2010 production rate in $75 \%$ of pathways [11], [12]. Regardless of whether the combustion emissions associated with this production can be mitigated by carbon capture and storage, eliminating upstream methane emissions is necessary to avoid the most severe effects of climate change.

State and federal governments throughout North America have enacted regulations in recent years to address methane emissions from oil and gas activity. California, Colorado, Pennsylvania and several other states now require periodic leak detection and repair (LDAR) programs at upstream and midstream facilities to find and fix leaks [11-15]. Separately, some oil and gas companies have also implemented voluntary LDAR programs to reduce methane leakage from their operations [18]. The most common technologies approved by regulators and used in these LDAR programs include EPA's Method-21 and optical gas imaging (OGI) based infrared cameras. Recent field work has shown that these OGI-based LDAR surveys have been effective in reducing emissions over several years [18]. Despite this success, there are challenges in scaling OGI-based LDAR to achieve rapid emission detection across vast geographic and temporal scales.

OGI surveys require an operator to manually inspect every potential leak source. Existing LDAR requirements typically specify one to four OGI surveys per year. The efficacy of these programs is limited by the probability that large unintended emissions (referred to as fugitive emissions or leaks) will persist for many months before detection. Ensuring that large emitters are quickly found and addressed therefore requires frequent LDAR surveys. However, frequent OGI-based LDAR surveys across thousands of sites quickly become logistically challenging and cost prohibitive.

Recently, several companies have developed novel approaches to methane leak detection that address the survey frequency limitation of OGI surveys [19]. Based on publicly available information, we can define three broad classes of new detection methods:

1. Novel component or equipment-level survey methods: OGI and EPA Method 21 surveys inspect every component and identify the source of emissions as part of the inspection. Drone- and some truck- and plane-based platforms provide similar specificity at 
potentially higher survey speed and lower cost. Technologies in this class were tested during the Stanford/EDF mobile monitoring challenge [19].

2. Site level screening methods: Rapid site-level screening may be used to identify high emitting sites that warrant component-level secondary follow up surveys. Site-level screening techniques were also tested in the mobile monitoring challenge and deployed in numerous academic studies [19], [20].

3. Continuous monitoring methods: Sensors are permanently installed in proximity to oil and gas sites and trigger follow up surveys when they detect an anomalous emission. Like site-level screening programs, continuous monitors allow rapid detection of large emissions while reducing the number of components that must be inspected directly.

Regulators and operators require a method for comparing the emissions reduction effectiveness of LDAR programs using continuous monitoring and site- or equipment-level screening methods to that of conventional LDAR programs. For example, Colorado's methane regulations require periodic leak detection surveys using a handheld OGI camera or an equivalent technique [21]. However, the method for determining whether a technique is equivalent is not specified. This is referred to as 'technology equivalence'.

A recent framework on technology equivalence developed jointly by scientists, industry experts, and regulators emphasizes the role of models in comparing the performance of different technologies and methods [22]. These models help evaluate new LDAR programs without the need for expensive, time-consuming, and concurrent field-trials with new technologies.

In this work, we explore the equivalence of novel LDAR programs to conventional OGI-based LDAR programs, demonstrate a model-based equivalence analysis, and provide recommendations for cost-effective emissions mitigation policies. We examine the trade-offs in survey speed, spatial resolution, and emissions mitigation between site-level and componentlevel surveys using the Fugitive Emissions Abatement Simulation Toolkit (FEAST) [23]. FEAST represents dynamic emissions from a gas field through time and models the emissions mitigation resulting from LDAR programs. The results demonstrate that a higher frequency site level screening survey coupled with a component level survey for repair may result in greater emission reductions than a lower frequency component level survey without increasing costs. Critically, we show that there is no one-size-fits-all approach to technology choice: emissions mitigation is strongly affected by survey frequency, leak occurrence rates, and emissions size distribution. Our approach illustrates how FEAST can provide the modelling framework required to evaluate equivalency between disparate LDAR programs [22]. All model code and associated documentation is made publicly available as part of this publication for use by scientists, operators, and regulatory agencies.

\section{Methods}

FEAST combines a stochastic model of methane emissions at upstream oil and gas facilities with a model of leak detection and repair (LDAR) programs to estimate the efficacy and cost of LDAR programs [23]. All simulation settings used in this work are further documented in the supporting information (SI sections S2 and S3). A detailed description of the underlying model construction can be found in [24]. 


\subsection{Facility Descriptions - Activity Factors}

Effective representation of methane emissions from upstream facilities requires both activity factors and emission characteristics corresponding to specific oil and gas basins. In this work, we use publicly available data from the U.S. EPA Greenhouse Gas Reporting Program (GHGRP) and the Colorado Oil and Gas Conservation Commission (COGCC) to create an activity model representative of sites in the Denver-Julesburg (DJ) basin [24-25]. On average, there are 1.9 wells per site in the DJ-basin, with a range between 1 and 51 wells per site. Activity data for this work also include component counts and frequency of unloading events (SI section S2).

\subsection{Emissions Descriptions - Emissions Factors}

FEAST simulates vents and fugitive emissions. Vents are emissions that occur by design, such as emissions from gas-driven pneumatic devices, and pressure-release valves. We also model liquid unloading events. For this work, unloading events are represented based on the total number of events and emissions reported to the GHGRP [25], while all other vents are approximated by drawing emission rates from an empirical distribution of observed emissions.

The fugitive emission model is characterized by an empirical emissions distribution and a leak production rate. FEAST simulates new leaks as independent random events in a Poisson process. The leak production rate is estimated based on the number of emissions found in repeated surveys of production equipment including tanks, pneumatics, and fugitive equipment under Colorado's OGI survey regulations [16], [27]. The empirical emission dataset is compiled from component level emission measurements from five recent publicly available studies [18], [28][31]. The studies included here did not distinguish between vents and leaks. In this work we assume that $46 \%$ of emissions simulated from the dataset are vents (see SI section S4.4 for additional detail). LDAR programs do not affect vented emissions in the simulation, but vents can cause site level surveys to trigger follow up actions at sites without significant fugitive emissions. The emission rate for each emission is drawn with replacement from the dataset. This approach is preferred compared to standard EPA emission factors approach because of the importance of super-emitters and skewed emissions distributions on the mitigation outcomes of LDAR programs. Additional information describing the data is available in SI section S2.

Several prior studies have demonstrated the highly skewed nature of methane emissions, with the top $5 \%$ of sites contributing to between $20 \%$ and $70 \%$ of total emissions depending on the geologic basin surveyed [35]-[38]. In a sensitivity analysis, we use a parametric emission size distribution to vary the contribution of the largest emitters to total emissions to understand how variability between basins will affect mitigation outcomes. The parametric distribution was defined such that emissions from the $80^{\text {th }}$ percentile and larger were drawn from a power law distribution rather than the empirical distribution. The exponent characterizing the power law was then adjusted to achieve a range of skews in the emissions distribution as observed in field campaigns throughout North America. The parameterization maintains the median emission rate while exploring the range of equivalency conditions under different emission distributions.

\subsection{Model Simulation:}

Every FEAST run simulates undirected inspection and maintenance (UDIM) activities in addition to LDAR programs.. The UDIM model represents typical maintenance activities undertaken by operators. The UDIM model causes the total number of emissions to equilibrate 
over time in the absence of an LDAR program as UDIM repairs offset the occurrence of new fugitive emissions. The LDAR models simulate regulatory LDAR surveys that occur in addition to UDIM activities. Comparing emissions in a UDIM-only scenario to an LDAR program helps calibrate the model by comparing model derived emissions reduction from OGI-based LDAR surveys to recent field data and regulatory models [18], [21]. The sensitivity of results to variations in the assumed UDIM repair rate are explored in SI section S4.

\subsection{LDAR Programs:}

In this study, we simulate two types of LDAR programs: component-level detection programs and tiered detection programs. Component level detection programs evaluate every component for emissions independently and identify the source of emissions at the time of detection. Tiered detection methods take a hybrid approach to leak detection: an initial survey to perform site-level screening, followed by a second component-level survey to identify components for repair at high-emitting sites.

\subsubsection{Component-level survey:}

OGI camera surveys are an example of a component level survey. Different component level survey methods are distinguished by their probability of detection (PoD) curves, survey speed, and cost as shown in Table 1. The median detection limit is defined as the leak size at which the probability of detection is $50 \%$. Several recent empirical, peer-reviewed performance assessment studies are used to parameterize and validate the PoD curves [19], [27], [32]. Leaks detected by a component level survey are immediately passed to the repair process which eliminates the leak one day later.

\subsubsection{Tiered surveys:}

Tiered detection programs use a screening method to identify production sites with high emission rates, similar to several existing plane-based technologies [20]. Like the componentlevel detection model, the probability of detection curve is modeled as a sigmoid based on empirical observations in recent peer-reviewed studies (SI section S3) [19]. For these simulations, all sites with emissions that are detected by the screening method are flagged for follow up by an OGI camera inspection to identify the source(s) of the emissions. Table 1 shows the key parameters used in the OGI-based (component-level) and plane-based (tiered) LDAR programs.

\section{Table 1 Key parameters used to specify detection methods.}

\begin{tabular}{|l|l|l|l|}
\hline & $\begin{array}{l}\text { Median detection limit } \\
(\mathrm{kg} / \text { day })\end{array}$ & Survey speed & $\begin{array}{l}\text { Cost } \\
(\$ / \text { site })\end{array}$ \\
\hline OGI & 2 & 6 sites/day & $600 \$ /$ site \\
\hline Plane & 94 & 222 sites/day & $100 \$ /$ site \\
\hline
\end{tabular}

\subsection{Simulation settings}

Simulations represent emissions from 100 well-sites over three years. The simulations have a time resolution of one hour and 300 Monte Carlo iterations were completed for every LDAR program and emission scenario represented in this work. 
The concept of technology equivalence is central to incorporating new technologies in regulatory LDAR programs [22]. While definitions vary across jurisdictions, it is typically defined in terms of mitigation outcomes - if two leak detection methods under separate LDAR program parameters achieve similar emissions mitigation, they are said to be equivalent. We present a series of results that evaluate equivalency between LDAR programs with increasing degrees of freedom and implications for the cost-effectiveness of methane mitigation.

\subsection{Emission mitigation under OGI and tiered LDAR programs}

Figure 1 shows the result of FEAST simulations for an upstream O\&G basin with 100 well-sites under three different LDAR scenarios - UDIM, OGI survey, and a tiered program. The tiered program consists of an aerial screening survey with OGI follow up referred to as Plane + OGI. All surveys represented in Figure 1 are conducted semi-annually. The first of the two surveys start on day one in these simulations.

Figure 1.A shows the first 30 days of emissions during a single Monte Carlo iteration of FEAST for UDIM, OGI and Plane + OGI scenarios. Since LDAR programs only affect leaks, vented emissions are identical across all three scenarios. Unloading events result in the short duration spikes that drive the emission rate to over $20 \mathrm{~kg} / \mathrm{day}$ per well. Figure 1.A shows that the rapid survey speed of the tiered detection method allows emissions to be found more quickly than a traditional OGI survey. However, the OGI method surpasses the Plane + OGI program by the end of the thirty-day period due to the lower detection threshold of the OGI survey in comparison to the preliminary aerial survey.

Figure 1.B extends the time series from Figure 1.A over the full three-year duration of the simulation. The time series shows the daily average emission rates. With a higher detection threshold than the OGI camera, the plane-based survey identifies fewer sites with emissions compared to OGI. Thus, fewer sites are flagged for follow up repair, resulting in higher average emissions when the two methods have the same survey frequency.

Figure 1.C shows the emissions mitigation achieved under both LDAR programs, relative to emissions in the UDIM scenario. A semi-annual OGI-based LDAR survey results in fugitive emissions mitigation of approximately $60 \%$, similar to EPA's assumptions in its methane regulations [33]. By comparison, the Plane + OGI LDAR program achieves emissions mitigation of about $33 \%$ less than the conventional OGI survey. In this scenario, the two LDAR programs are not equivalent. The error bars represent variability from 300 Monte-Carlo iterations of the LDAR programs. Although FEAST models detection as a probabilistic process, the uncertainty range shown in Figure 1.C is driven by variability in the emission simulation rather than the detection simulation (See SI Figure S9). Therefore, the relative performance of the two simulated LDAR programs to each other is more certain than the absolute emissions in either case.

Figure 1.D shows the range of mitigation costs incurred by the OGI and Plane + OGI programs across the same 300 Monte Carlo iterations as Figure 1.C. Although the Plane + OGI program achieves less mitigation than the conventional OGI program, it does achieve a lower cost per ton of avoided $\mathrm{CO}_{2}$ equivalent emissions. The mitigation cost for the Plane + OGI program is $\$ 11 / \mathrm{t}$ 
266 CO2e, about $31 \%$ lower than the $\$ 16 / t$ CO2e cost for OGI-based mitigation. In this example, the 267 Plane survey flagged just $10 \%$ of sites for follow up surveys.
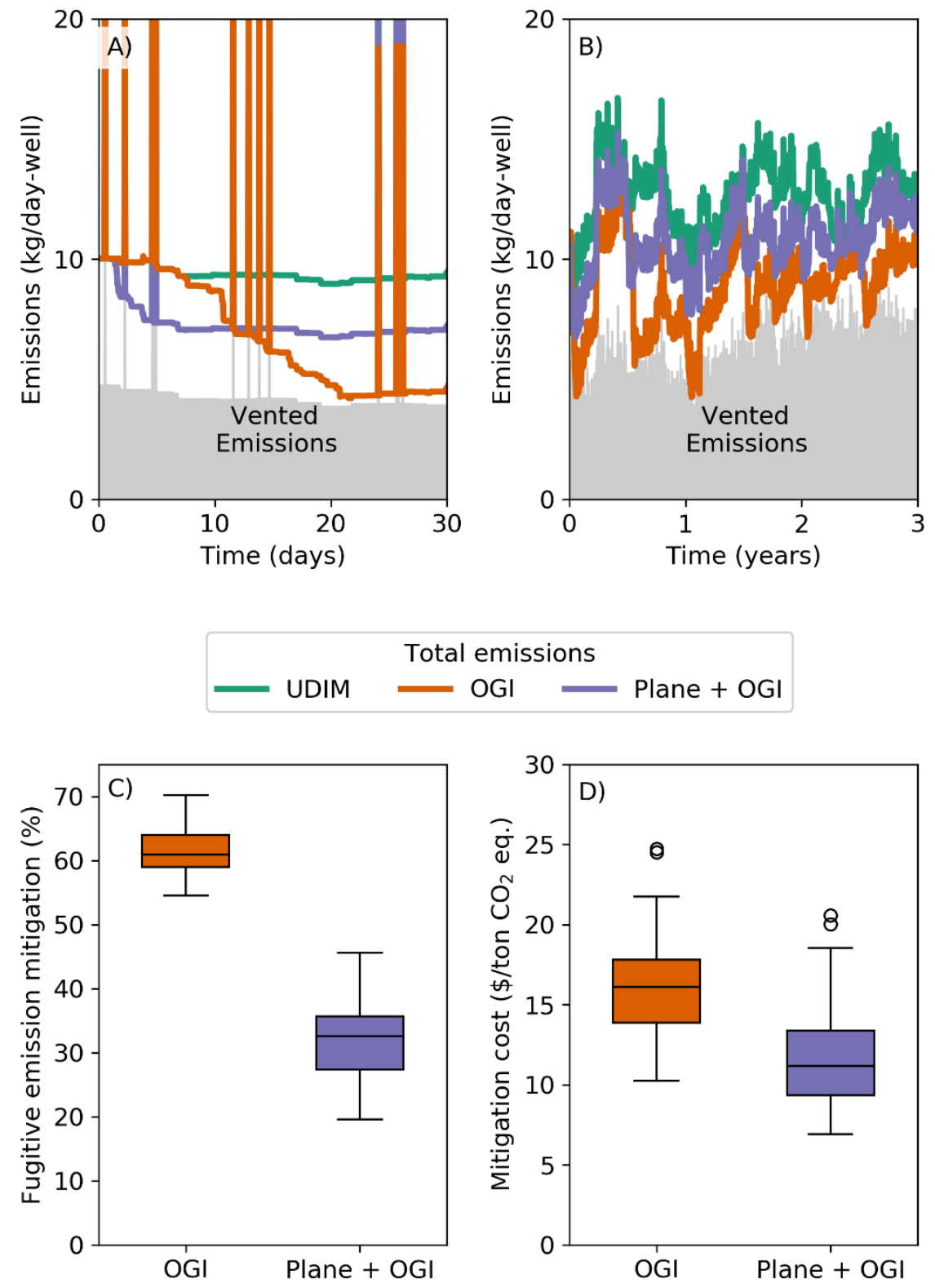

Figure 1. Results of FEAST simulations representing OGI surveys ("OGI") and plane-based screening with OGI follow up ("Plane + OGI") at high emitting sites A.) 30 days of hourly emissions in a single realization generated by FEAST B.) One-day moving average emission rate from a single realization under three LDAR scenarios over the entire simulation period of 3 years C.) Distribution of mitigation achieved by OGI and Plane+OGI LDAR programs D.) Distribution of mitigation costs for the OGI and Plane+OGI LDAR programs. Outliers are greater than the $75^{\text {th }}$ percentile by more than 1.5 times the interquartile range. 
3.2 Mitigation equivalence dependence on survey frequency

277 Since a plane-based survey will not detect as many emissions as an OGI survey, the Plane + OGI 278 program must survey more frequently to achieve equivalent emissions. Figure 2 shows the impact of survey frequency on the mitigation and cost of the two LDAR programs.

Figure 2.A compares the component level and site level emission rate distributions under UDIM conditions to the median detection thresholds of the OGI and Plane technology (see SI Figure S2 for additional details of the PoD curve). Overall, 94\% of emissions come from sources larger than the median detection threshold of the OGI camera. However, only $41 \%$ of emissions come from sites with a total emission rate greater than the median detection limit of the Plane technology.

Figure 2.B shows the emissions mitigation achieved through both LDAR programs as a function of survey frequency. For the conventional OGI-based survey, increasing survey frequency from two to four times per year increases mitigation from $60 \%$ to $73 \%$. This is similar to the emissions mitigation expected in federal regulations, where semi-annual and quarterly surveys reduce emissions by $60 \%$ and $80 \%$, respectively [33]. Thus, the model parameters here reproduce the emissions mitigation current regulations expect to be achieved under different OGI-based LDAR survey frequencies.

Increasing survey frequency reduces the duration of fugitive emissions. In the UDIM scenario, leaks have an average duration of 208 days. Under an LDAR program, leaks that are large enough to be detected will have an average duration of approximately one-half the time between surveys: for example, quarterly surveys result in an average duration of approximately 45 days for large leaks. LDAR programs mitigate emissions by reducing their duration.

Consider a mitigation target of $40 \%$ reduction in fugitive emissions. The conventional OGIbased LDAR survey can achieve this mitigation target with an annual survey. Equivalently, the tiered Plane + OGI LDAR program achieves $40 \%$ mitigation if the survey frequency is increased to approximately 3 surveys per year. Higher levels of mitigation can be achieved with either program if the survey frequency is increased further, although the plane-based survey cannot achieve $80 \%$ mitigation even with monthly surveys due. While increasing the survey frequency decreases the duration of detected emissions, emissions much smaller than the detection threshold remain unaffected even at high survey frequencies. The detection threshold of a screening technology thus places an effective upper bound on the amount of mitigation that can be achieved. 


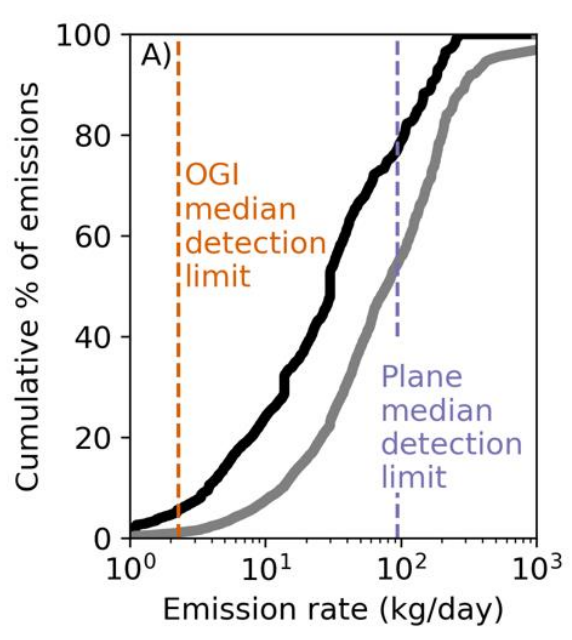

Emission distributions Component Site

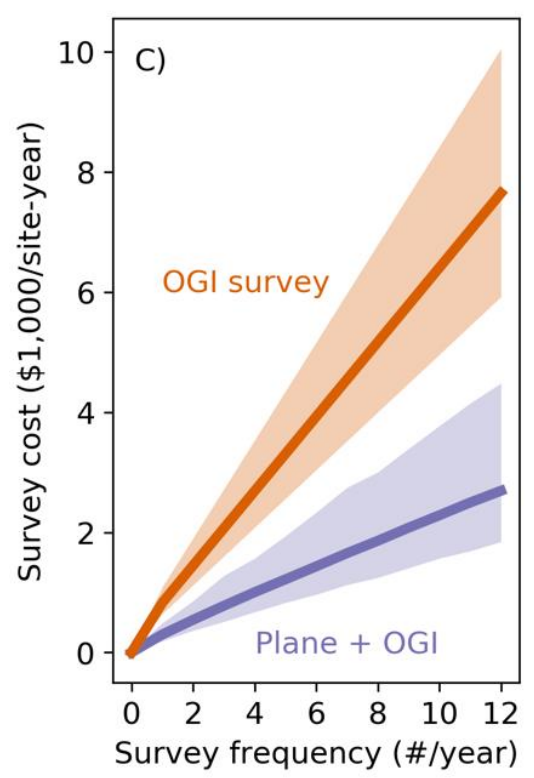

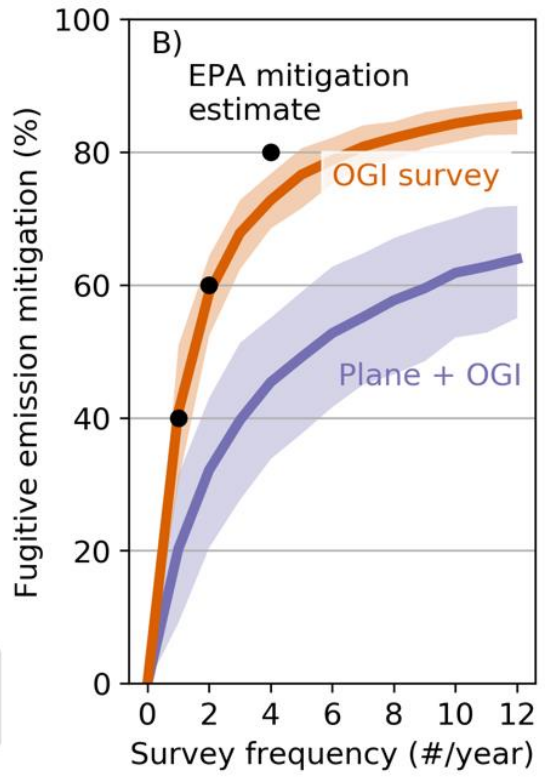

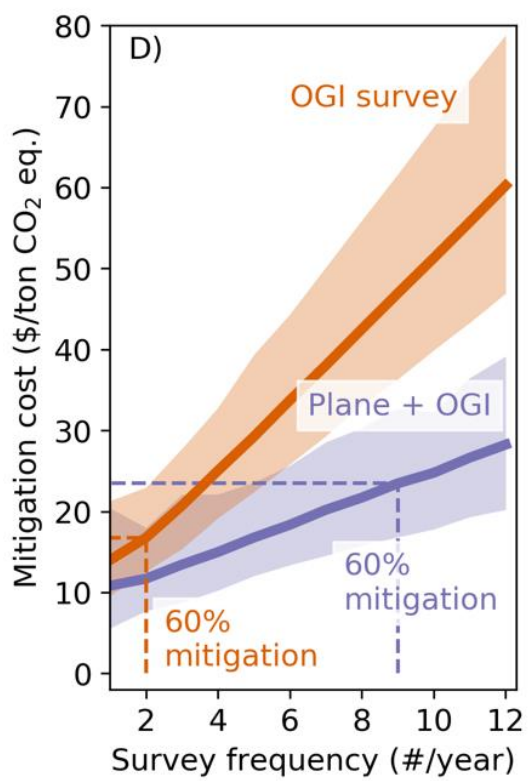

Figure 2. LDAR simulation results for an OGI detection threshold of $2 \mathrm{~kg} /$ day and a plane detection threshold of $94 \mathrm{~kg} /$ day A.) Component-level and site-level cumulative emission distributions with probability of detection curves for the simulated Plane and OGI detection methods. A-C) Fugitive emissions mitigation, survey cost and mitigation cost with OGI and Plane + OGI LDAR programs over a range of survey frequencies. Uncertainty ranges represent the $95 \%$ confidence interval generated by Monte Carlo iterations.

Figure 2.C shows that the cost of surveys for each LDAR program is proportional to the survey frequency. Prior studies have shown that the majority of costs associated with implementation of LDAR programs are reflected in the survey costs [34]. The US EPA's own analysis of its methane regulations show that semi-annual OGI-based LDAR surveys contributes to over $70 \%$ of the total cost of the LDAR program. The simulations shown in Figure 2.B-C suggest $60 \%$ fugitive emission reduction using either semi-annual OGI surveys or 9 plane-based screening surveys per year with OGI follow up. Under our cost assumptions, semi-annual OGI surveys 
incur costs of $\$ 1400 /$ site-year compared to $\$ 2000 /$ site-year to achieve equivalent mitigation with more frequent Plane + OGI surveys.

The results of Figure 2.B and 2.C were combined to generate Figure 2.D: the cost per metric ton of $\mathrm{CO}_{2}$ equivalent emissions mitigated. The nonlinear mitigation curve of Figure $2 . \mathrm{B}$ causes the mitigation cost to increase more slowly for survey frequencies less than 3/year: as survey frequency increases from zero, mitigation also increases partially offsetting the added survey costs. At higher survey frequencies, mitigation approaches its asymptote resulting in near linear growth in mitigation cost. The result illustrates that the marginal cost of mitigation increases as the survey frequency increases.

\title{
3.3 Cost-effectiveness of equivalent LDAR programs requires optimization across survey frequency and detection threshold.
}

The cost-effectiveness of emissions mitigation depends on both the leak detection method and the survey frequency. Here, we explore the cost-effectiveness of fugitive emissions mitigation $\left(\$ / \mathrm{t} \mathrm{CO}_{2} \mathrm{e}\right)$ by modeling two generic leak detection methods - component-level surveys at an average cost of $\$ 600 /$ site and site-level surveys at $\$ 100 /$ site. Follow up OGI surveys are charged at the same rate per component as the generic component-level surveys. While keeping these cost assumptions constant, Figure 3 illustrates how the mitigation cost changes depending on the detection threshold and survey frequency of LDAR programs.

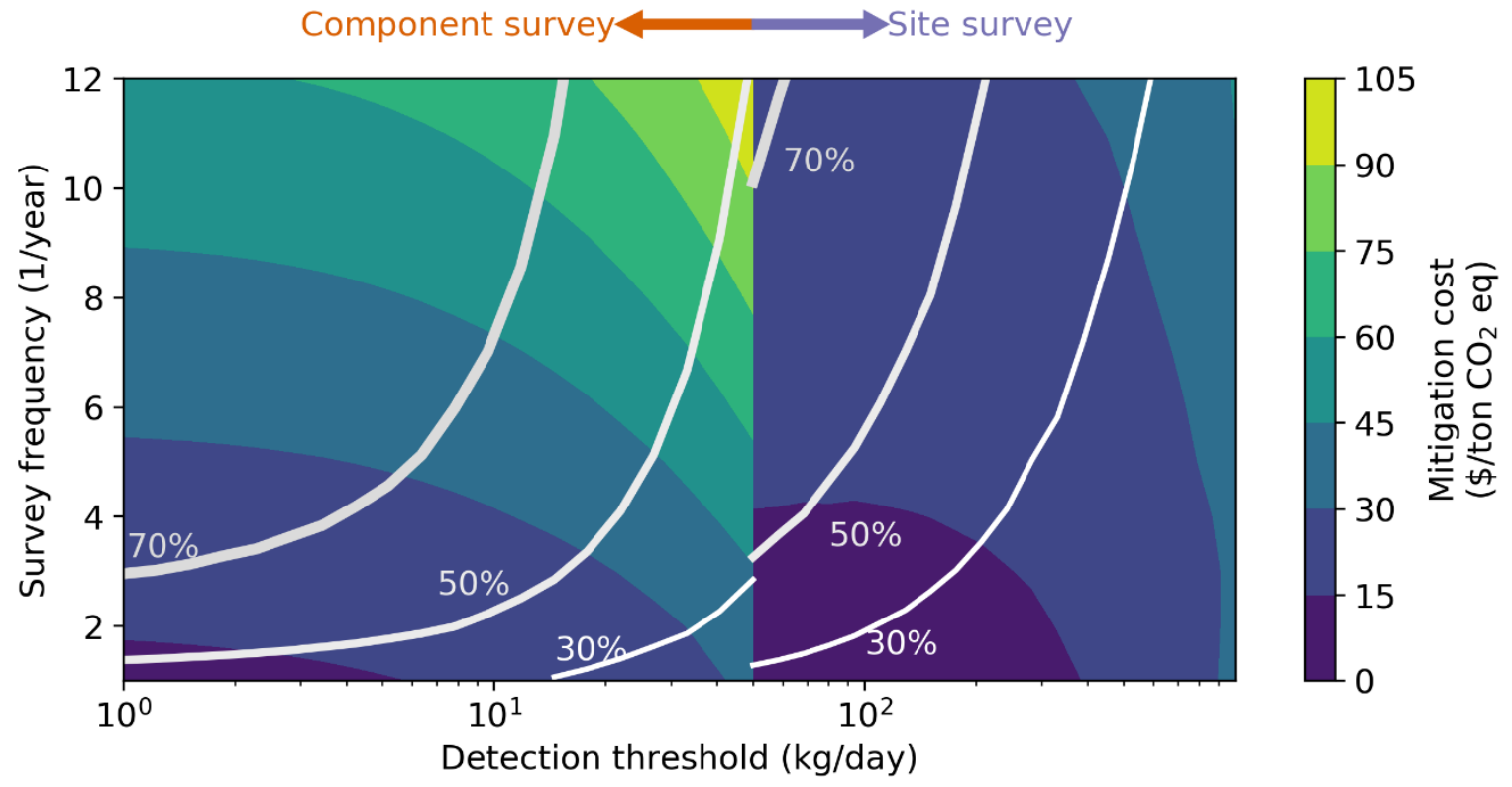

\begin{abstract}
Figure 3. $\mathrm{CO}_{2}$ equivalent mitigation cost of modeled technologies over a range of survey frequencies and detection thresholds. White contour lines indicate fugitive emissions mitigation percentages with the line thickness proportional to mitigation level, while the color map indicates mitigation cost.
\end{abstract}

Methods with detection thresholds above $50 \mathrm{~kg} /$ day were modeled as tiered detection programs while methods with detection thresholds less than $50 \mathrm{~kg} /$ day were modeled as component-level surveys. Constant-mitigation contours are indicated by white curves. For example, the curves labeled $70 \%$ indicate all combinations of detection sensitivity and survey frequency that result in $70 \%$ mitigation of fugitive emissions. The location with the lowest cost along a mitigation 
contour indicates the cost-optimal mitigation strategy for a particular mitigation target under these assumptions.

Horizontal transects across the mitigation contours reveal the impact of increasing detection threshold while holding the survey frequency constant. For small detection thresholds between 1 $\mathrm{kg} / \mathrm{d}$ and $10 \mathrm{~kg} / \mathrm{d}$ (high sensitivity), there is little change in mitigation as sensitivity increases because small emitters account for a small fraction of total emissions. However, as the detection threshold exceeds $10 \mathrm{~kg} / \mathrm{d}$, mitigation is more sensitive to detection threshold. Thus, while increasing sensitivity of detection technology can improve mitigation outcomes, the marginal improvement in sensitivity below about $10 \mathrm{~kg} / \mathrm{d}$ does not result in a corresponding increase in emissions mitigation. One can therefore trade high sensitivity for lower cost without adverse mitigation outcomes.

Considering the color map of Figure 3 reveals trends in mitigation cost. Continuing with the example site level detection threshold of $94 \mathrm{~kg} /$ day, the mitigation cost is $11 \$ / \mathrm{t} \mathrm{CO}_{2} \mathrm{e}$ for a survey frequency of $2 /$ year but increases to $22 \$ / t \mathrm{CO}_{2}$ e for a survey frequency of 8/year. In addition, mitigation cost increases as detection threshold increases. This trend occurs because the cost per component or site surveyed is independent of sensitivity in this simulation. The survey cost of the component-level programs remains constant while the total mitigation decreases, resulting in an overall increase in mitigation cost. By contrast, the costs of the tiered programs decline as the detection threshold increases because fewer sites are flagged for follow up surveys. However, the results show that the decrease in cost due to follow up surveys is not sufficient to offset the decline in mitigation caused by increasing the detection threshold.

Tiered detection programs must efficiently direct ground crews to achieve sufficient emissions mitigation without incurring secondary survey costs that exceed the savings achieved by the site level survey. Tiered methods that identify high emitting equipment rather than sites may be more successful if they can significantly reduce the time on site required of ground crews and avoid misallocating ground crews due to vented emissions.

Our results also show that tiered detection programs are more cost effective if the mitigation goal is less stringent. For example, Figure 3 shows tiered methods with a site level detection threshold up to $300 \mathrm{~kg} /$ day can achieve $30 \%$ mitigation with less than 4 surveys per year more cost effectively than annual component level surveys but are less competitive if the mitigation target is increased to $70 \%$. Similarly, a site-level technology with a detection threshold of $60 \mathrm{~kg} / \mathrm{d}$ can achieve $50 \%$ mitigation with quarterly surveys at a cost of $\$ 15 / \mathrm{tCO} 2 \mathrm{e}$, lower than the equivalent semi-annual OGI-based LDAR survey cost of \$17/tCO2e. The results from Figure 3 are sensitive to the underlying emission rate distribution as described in the following section.

\section{4 "Equivalence" depends on the natural gas basin where a technology is applied.}

The skew of an emission distribution affects equivalence between LDAR programs. An LDAR program that specializes in quickly identifying large leaks will perform better if emission distributions are more skewed, because high-emitting sites will account for a greater fraction of total emissions. Conversely, a component level method that surveys less frequently but has a more sensitive detector will achieve a better mitigation fraction in less skewed distributions because it will not allow midsize leaks to persist indefinitely. While Figures 1-3 rely on the 
empirical emission distribution compiled for this work, this section explores how equivalence is sensitive to changes in the emission distribution.

Figure 4 shows the technology detection threshold required to achieve a target emissions mitigation level across different emission distributions. The orange and purple curves represent mitigation under component level and tiered detection programs, respectively. In all cases, the survey frequency was set to 6 surveys per year, while detection threshold was varied to achieve the target emission mitigation rate.

In a highly skewed emission distribution as observed in the Uintah or Marcellus basin, 50\% mitigation can be achieved with a tiered detection program that has a detection threshold of 200 $\mathrm{kg} /$ day. However, a detection threshold of $50 \mathrm{~kg} /$ day would be required to achieve the same level of mitigation in a less skewed distribution as observed in Medicine Hat in Alberta. More skewed distributions allow the same mitigation targets to be achieved with a higher detection threshold, resulting in a positive slope for all tiered and component surveys modeled in Figure 4.

The vertical gray lines show results from empirical studies conducted in the last five years from U.S. and Canadian shale basins. Due to the sample size in these studies, the uncertainty in the fraction of emissions from the top $5 \%$ of emitters may be large compared to the variability between basins. The range of skew measured in various basins shown in Figure 4 give an indication of the combined uncertainty and variability that exists in emission distributions. Furthermore, the distribution of emissions that occur in a particular basin may evolve over time due to maturing infrastructure, new wells, and production decline. An alternative LDAR program may become more or less effective in comparison to OGI over time.
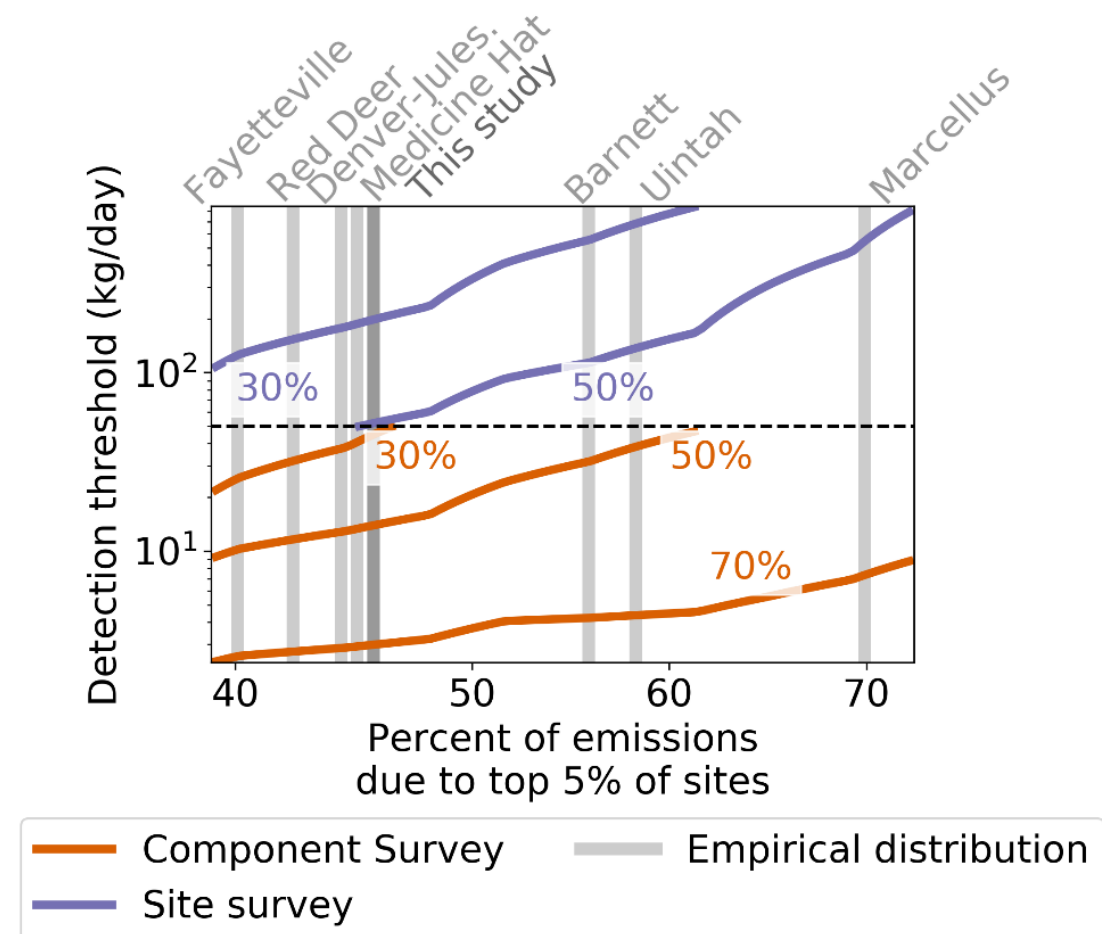

Figure 4. The effect of emission size distribution on the detection threshold required to achieve a given mitigation target. Purple and orange curves indicate the detection threshold required to achieve mitigation for component and site level surveys, respectively. Follow up survey sensitivity is kept constant for all site 
survey methods. Grey bars indicate the emission distribution skew observed in eight empirical studies of site level emissions.

\section{2}

\section{Discussion and Study Limitations}

According to the EPA greenhouse gas inventory, more than 5 million tons of methane leaked from US natural gas infrastructure in 2018 [1]. New mobile and fixed-sensor technologies could provide a cost-effective approach to reduce emissions. Yet, regulatory approval of these new methods critically depends on a demonstration of equivalence to existing LDAR approaches. The equivalence analysis described here provides the modeling framework required to quantitatively compare LDAR programs while also highlighting the sensitivity of results to the underlying emission model.

Equivalent emissions mitigation can be achieved with a broad range of sensitivities by choosing the appropriate survey frequency and/or using a tiered detection approach. Tiered detection approaches take advantage of the heavy-tailed nature of emission distributions to allocate resources to the largest emissions, while component level surveys invest the same amount of time in identifying emitters of all sizes. Tiered approaches must be efficient in dispatching ground crews to offset the additional costs from increased survey frequencies.

Depending on their approach, LDAR programs will be affected differently by the emission size distribution. While the composite emission distribution used in this work falls within the range of emission distributions that exist in the US, Figure 4 shows that no distribution can accurately represent all basins. Furthermore, the uncertainty in the tail of the component-level emission distribution remains an important source of uncertainty in mitigation modeling. Accurately representing mitigation requires improved measurements of emission distributions.

The "leak production rate" also remains a critical source of uncertainty in mitigation modeling. While many studies have captured "snap shots" of the state of emissions in gas fields, much less work has been done to repeatedly survey the same sites and evaluate the rate at which new emissions occur. To separately evaluate the mean time to failure and the effect of undirected maintenance activities, the same sites must be surveyed frequently, and emitters must be tracked through time.

Additional empirical data would increase the confidence in equivalence assessments. The sensitivity analysis presented in SI section S4 suggests that improving precision in the leak production rate estimate will decrease uncertainty the most, followed by developing basinspecific emission distributions. Coupling FEAST with a process-based model similar to that described by Cardoso-Saldon et al. [39] would provide a more accurate model of vents and further reduce uncertainty.

We draw the following broad conclusions from the results of this work that can aid oil and gas operators and regulatory agencies in developing LDAR programs using new methane detection technologies:

1. Equivalent emissions mitigation can be achieved by LDAR programs with different detection thresholds by varying the survey frequency. 
2. Median detection threshold of new technologies, to first order, present effective lower and upper bounds for emissions mitigation. At the lower end, decreasing the detection threshold below $10 \mathrm{~kg} / \mathrm{d}$ does not increase mitigation outcomes proportionally because of skewed leak-size distributions. At the upper end, emissions mitigation with high median detection threshold technologies does not increase in proportion to survey frequency as emissions smaller than the detection threshold remain unaffected even at high survey frequencies.

3. Vented emissions play a critical role in the cost-effectiveness of tiered detection programs that direct ground crews based on site-level emissions detection. Without a reliable way to differentiate sites with high vented emissions from those with high fugitive emissions, tiered programs risk directing ground crews to many sites with little mitigation benefit, thereby increasing costs.

4. The survey frequency and detection threshold required for equivalent emission mitigation will depend on the emission size distribution in the basin where the LDAR program is applied. Evaluation of the efficacy of LDAR programs and technology equivalence periodically to account for (a) changes to emission-size distribution, and (b) reduction in emissions over time will be critical to ensure mitigation targets are achieved throughout the duration of the program.

New methane detection technologies and platforms - continuous and survey-based - represent an opportunity to cost-effectively address methane emissions from the oil and gas industry. The degrees of freedom in LDAR program parameters such as technology choice, hybrid detection, survey frequency, and detection threshold provide a method to design methane mitigation policies that best tackle issues specific to the gas field or operator. As states and countries around the world converge on methane emissions as a cost-effective, near-term approach to address climate change, FEAST is a quantitative tool to for assessing new technologies, evaluating the outcomes of mitigation programs, and achieving methane mitigation targets. Future work on this model will seek to enable the evaluation of satellite technologies and continuous monitoring systems to provide a near real-time monitoring of methane emissions across the world. In light of the potential use of this model in regulatory rule making, all model code and documentation are made publicly available as part of this publication, including any future updates.

\section{Acknowledgements}

The authors would like to thank Daniel Zimmerle and Clay Bell at Colorado State University for numerous scientific discussions on methane emissions modeling during our work on this manuscript. The authors also acknowledge funding from Harrisburg University of Science and Technology and the Path to Equivalence grant with Colorado State University Award Number G-31051-01. 
References:

[1] U.S. Environmental Protection Agency, "Inventory of U.S. Greenhouse Gas Emissions and Sinks: 1990-2018 - EPA 430-R-20-002," Apr. 2020. Accessed: Aug. 13, 2020. [Online]. Available: https://www.epa.gov/ghgemissions/inventory-us-greenhouse-gasemissions-and-sinks.

[2] G. Myhre et al., "Chapter 8: Anthropogenic and Natural Radiative Forcing," in Climate Change 2013: The Physical Science Basis. Contribution of Working Group I to the Fifth Assessment Report of the Intergovernmental Panel on Climate Change, T. F. Stocker, D. Qin, G.-K. Plattner, M. Tignor, S. K. Allen, J. Boschung, A. Nauels, Y. Xia, V. Bex, and P. M. Midgley, Eds. Cambridge, United Kingdom and New York, NY, USA, 2013, pp. 659-740.

[3] D. J. Zimmerle et al., "Methane Emissions from the Natural Gas Transmission and Storage System in the United States," Environ. Sci. Technol, vol. 49, p. 54, 2015, doi: 10.1021/acs.est.5b01669.

[4] R. A. Alvarez et al., "Assessment of methane emissions from the U.S. oil and gas supply chain," Science (80-. )., 2018, doi: 10.1126/science.aar7204.

[5] A. Brandt et al., "Methane Leaks from North American Natural Gas Systems," Science (80-. )., vol. 343, pp. 733-735, 2014.

[6] R. W. Howarth, "Ideas and perspectives: is shale gas a major driver of recent increase in global atmospheric methane?," Biogeosciences, vol. 16, pp. 3033-3046, 2019, doi: 10.5194/bg-16-3033-2019.

[7] I. A. G. Wilson and I. Staffell, "Rapid fuel switching from coal to natural gas through effective carbon pricing," Nat. Energy, vol. 3, no. 5, pp. 365-372, May 2018, doi: 10.1038/s41560-018-0109-0.

[8] K. Tanaka, O. Cavalett, W. J. Collins, and F. Cherubini, "Asserting the climate benefits of the coal-to-gas shift across temporal and spatial scales," Nat. Clim. Chang., vol. 9, no. 5, pp. 389-396, 2019, doi: 10.1038/s41558-019-0457-1.

[9] X. Zhang, N. P. Myhrvold, Z. Hausfather, and K. Caldeira, "Climate benefits of natural gas as a bridge fuel and potential delay of near-zero energy systems," 2015, doi: 10.1016/j.apenergy.2015.10.016.

[10] A. L. Rich and H. T. Orimoloye, "Elevated Atmospheric Levels of Benzene and BenzeneRelated Compounds from Unconventional Shale Extraction and Processing: Human Health Concern for Residential Communities," Environ. Health Insights, vol. 10, pp. 7582, 2016, doi: 10.4137/EHI.S33314.

[11] IPCC, Global warming of $1.5^{\circ} \mathrm{C}$ An IPCC Special Report on the impacts of global warming of $1.5^{\circ} \mathrm{C}$ above pre-industrial levels and related global greenhouse gas emission pathways, in the context of strengthening the global response to the threat of climate change,. 2018.

[12] D. Huppmann et al., "IAMC $1.5^{\circ} \mathrm{C}$ Scenario Explorer and Data hosted by IIASA." Integrated Assessment Modeling Consortium \& International Institute for Applied Systems Analysis, 2018, doi: 10.22022/SR15/08-2018.15429.

[13] "Canada's methane regulations for the upstream oil and gas sector," Government of Canada, 2018. https://www.canada.ca/en/environment-climate-change/services/canadianenvironmental-protection-act-registry/proposed-methane-regulations-additionalinformation.html (accessed Aug. 13, 2019). 
Non - peer reviewed pre - print submitted to EarthArXiv

[14] C. Ruiz-Funes, DISPOSICIONES ADMINISTRATIVAS DE CARÁCTER GENERAL QUE ESTABLECEN LOS LINEAMIENTOS PARA LA PREVENCIN Y EL CONTROL INTEGRAL DE LAS EMISIONES DE METANO DEL SECTOR HIDROCARBUROS. 2018.

[15] "Final Regulation Order Subarticle 13: Greenhouse Gas Emission Standards for Crude Oil and Natural Gas Facilities." California Code of Regulations, pp. 1-71, 2017.

[16] Control of Ozone via Ozone Precursors and Control of Hydrocarbons via Oil and Gas Emissions. 2018.

[17] Wyoming Administrative Rules Chapter Environmental Quality Dept. of Air Quality Chapter 8: Nonattainment Area Regulations. US, 2018.

[18] A. P. Ravikumar et al., "Repeated leak detection and repair surveys reduce methane emissions over scale of years," Environ. Res. Lett., 2020, doi: 10.1088/1748-9326/ab6ae1.

[19] A. Ravikumar et al., "Single-blind Inter-comparison of Methane Detection Technologies-Results from the Stanford/EDF Mobile Monitoring Challenge," 2019, [Online]. Available: https://doi.org/10.1525/elementa.373.

[20] T. A. Fox, T. E. Barchyn, D. Risk, A. P. Ravikumar, and C. H. Hugenholtz, "A review of close-range and screening technologies for mitigating fugitive methane emissions in upstream oil and gas," Environ. Res. Lett., vol. 14, no. 5, p. 053002, Apr. 2019, doi: 10.1088/1748-9326/ab0cc3.

[21] Control of Ozone via Ozone Precursors and Control of Hydrocarbons via Oil and Gas Emissions. 2019.

[22] T. A. Fox et al., "A methane emissions reduction equivalence framework for alternative leak detection and repair programs," Elem Sci Anth, 2019, doi: 10.1525/elementa.369.

[23] C. E. Kemp, A. P. Ravikumar, and A. R. Brandt, "Comparing Natural Gas Leakage Detection Technologies Using an Open-Source 'virtual Gas Field' Simulator," Environ. Sci. Technol., vol. 50, no. 8, 2016, doi: 10.1021/acs.est.5b06068.

[24] C. E. Kemp, A. P. Ravikumar, and A. R. Brandt, "Comparing Natural Gas Leakage Detection Technologies Using an Open-Source Virtual Gas Field Simulator," Environ. Sci. Technol, 2016, doi: 10.1021/acs.est.5b06068.

[25] U.S. Environmental Protection Agency, "Facility Level Information on Greenhouse Gases Tool," 2017. https:/ghgdata.epa.gov/ghgp/main.do\#/listFacilityForBasin/?q=Find a Facility or Location $\& \mathrm{bs}=540 \&$ fid $=\& s f=11001000 \& l o w E=-$ 20000\&highE=23000000\&g1=1\&g2=1\&g3=1\&g4=1\&g5=1\&g6=0\&g7=1\&g8=1\&g9=1 $\& \mathrm{~g} 10=1 \& \mathrm{~g} 11=1 \& \mathrm{~g} 12=1 \& \mathrm{~s} 1=0 \& \mathrm{~s} 2=0 \& \mathrm{~s} 3=0 \& \mathrm{~s} 4=0 \& \mathrm{~s} 5=0 \& \mathrm{~s} 6=0 \& \mathrm{~s} 7=0 \& \mathrm{~s} 8=0 \& \mathrm{~s} 9=1 \& \mathrm{~s} 1$ $0=0 \&$ (accessed Oct. 23, 2019).

[26] COGCC, "COGCC/Data Downloads/GIS/Well Surface Location Data (Updated Daily)/Well Spots (APIs)," Colorado Oil and Gas Conservation Commission, 2019. https://cogcc.state.co.us/documents/data/downloads/gis/WELLS_SHP.ZIP (accessed Jan. 13, 2020).

[27] A. P. Ravikumar, J. Wang, M. Mcguire, C. S. Bell, D. Zimmerle, and A. R. Brandt, "Good versus Good Enough? Empirical Tests of Methane Leak Detection Sensitivity of a Commercial Infrared Camera," Environ. Sci. Technol, vol. 52, 2018, doi: 10.1021/acs.est.7b04945.

[28] D. Allen et al., "Measurements of methane emissions at natural gas production sites in the United States David," Proc. Natl. Acad. Sci., vol. 110, no. 44, pp. 18025-18030, Sep. 2013, doi: 10.1073/pnas.1315099110. 
[29] “City of Fort Worth Natural Gas Air Quality Study,” Fort Worth, TX, 2011. [Online]. Available: http://fortworthtexas.gov/uploadedFiles/Gas_Wells/AirQualityStudy_final.pdf.

[30] J. Kuo, "Final project report estimation of methane emissions from the California Energy Commission," 2012. doi: 500-09-007.

[31] C. S. Bell et al., "Comparison of methane emission estimates from multiple measurement techniques at natural gas production pads," Elem Sci Anth, vol. 5, no. 0, p. 79, Dec. 2017, doi: 10.1525/elementa.266.

[32] D. Zimmerle, T. Vaughn, C. Bell, K. Bennett, P. Deshmukh, and E. Thoma, "Detection Limits of Optical Gas Imaging for Natural Gas Leak Detection in Realistic Controlled Conditions," Environ. Sci. Technol., vol. 54, no. 18, pp. 11506-11514, 2020, doi: 10.1021/acs.est.0c01285.

[33] "Oil and Natural Gas Sector : Standards for Crude Oil and Natural Gas Facilities: Background Technical Support Document for the Proposed New Source Performance Standards 40 CFR Part 60, subpart OOOOa." EPA, Chapel Hill, NC, 2016, [Online]. Available: https://beta.regulations.gov/document/EPA-HQ-OAR-2010-0505-7631.

[34] A. P. Ravikumar and A. R. Brandt, "Designing better methane mitigation policies: the challenge of distributed small sources in the natural gas sector," Environ. Res. Lett., vol. 23, no. 044023, 2017, doi: 10.1088/1748-9326/aa6791.

[35] A. M. Robertson et al., "Variation in Methane Emission Rates from Well Pads in Four Oil and Gas Basins with Contrasting Production Volumes and Compositions," 2017, doi: 10.1021/acs.est.7b00571.

[36] C. W. Rella, T. R. Tsai, C. G. Botkin, E. R. Crosson, and D. Steele, "Measuring Emissions from Oil and Natural Gas Well Pads Using the Mobile Flux Plane Technique," Environ. Sci. Technol, vol. 49, p. 4748, 2015, doi: 10.1021/acs.est.5b00099.

[37] M. Omara, M. R. Sullivan, X. Li, R. Subramanian, A. L. Robinson, and A. A. Presto, "Methane Emissions from Conventional and Unconventional Natural Gas Production Sites in the Marcellus Shale Basin,” 2016, doi: 10.1021/acs.est.5b05503.

[38] E. O'Connell et al., "Methane emissions from contrasting production regions within Alberta, Canada: Implications under incoming federal methane regulations," Elementa, vol. 7, no. 1, 2019, doi: 10.1525/elementa.341.

[39] F. J. Cardoso-Saldañ and D. T. Allen, "Projecting the Temporal Evolution of Methane Emissions from Oil and Gas Production Sites," Cite This Environ. Sci. Technol, vol. 54, pp. 14172-14181, 2020, doi: 10.1021/acs.est.0c03049. 


\section{New Technologies can Cost-effectively Reduce Oil and Gas}

2 Methane Emissions, but Policies will Require Careful Design to

3 Establish Mitigation Equivalence

\section{Contents}

13 Supplementary Information

New technologies can cost-effectively reduce oil and gas methane emissions, but policies will

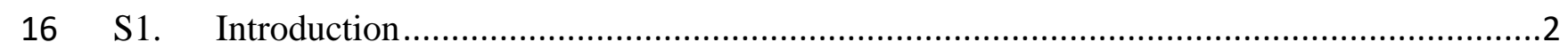

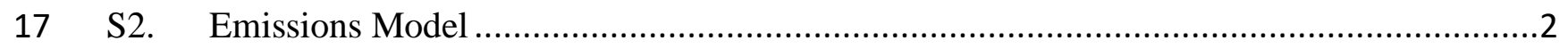

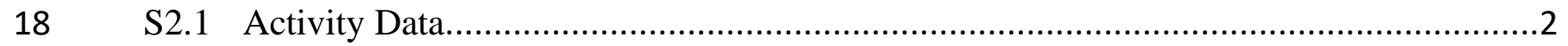

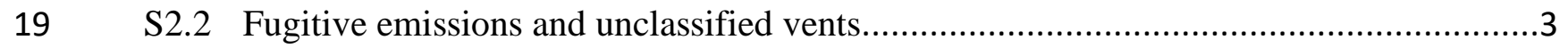

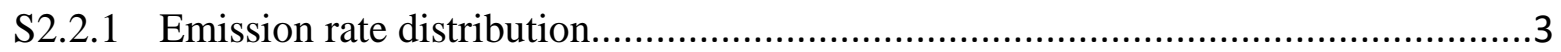

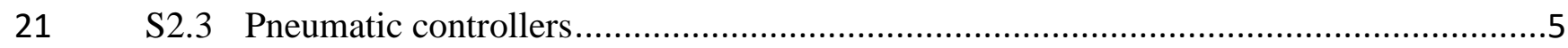

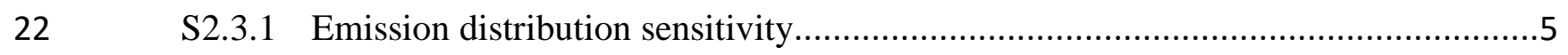

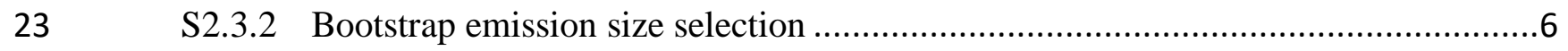

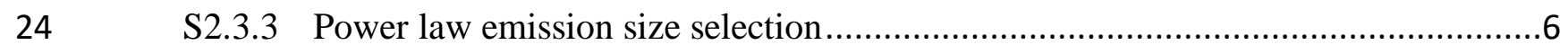

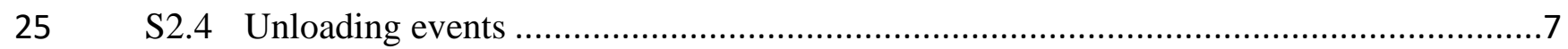

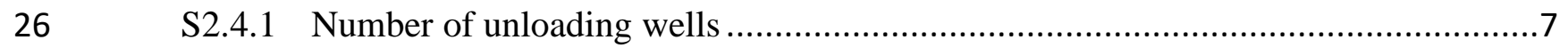

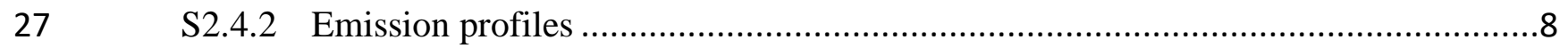

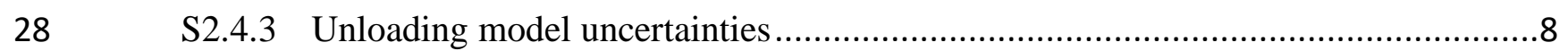

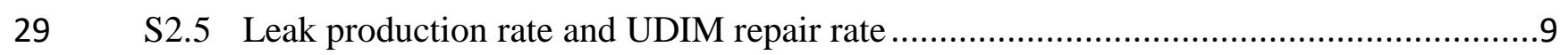

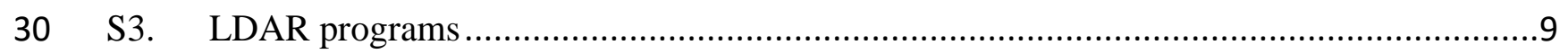

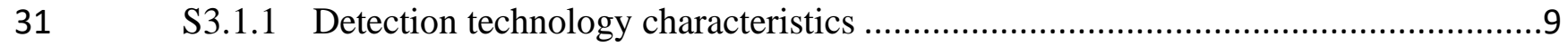

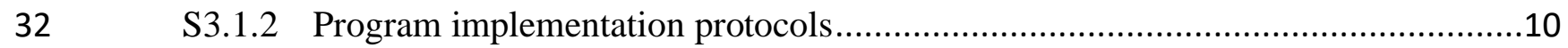


S3.1.3 Repair protocols

S3.2 Calculating mitigation costs

S4. Sensitivity Analysis .

S4.1 Emission rate distribution.

S4.5 Confidence in equivalence assessments

S1. Introduction

The results presented in the main text rely on the Fugitive Emission Abatement Simulation

Toolkit (FEAST). Section S1 is this introduction. The parameters used to specify emissions and LDAR programs in FEAST are further explained in sections S2 and S3, respectively. Section S4 presents a sensitivity analysis that describes how uncertainty in the input parameters impact results.

\section{S2. Emissions Model}

\section{S2.1 Activity Data}

FEAST represents production infrastructure as a hierarchy of components and sites. A simulation contains many sites, and a site contains many components. Every component can be a source of fugitive emissions, uncategorized vents, or unloading emissions.

Sites in this study are modeled after upstream well pads at unconventional oil and gas sites. Given the modular nature of the site representation, detailed activity data (equipment per site, components per equipment) can be used to represent any oil and gas facility, thereby expanding the potential use for the model. Each site is assigned a fixed number of wells drawn with replacement from the population of well pads in Colorado. The Colorado Oil and Gas Conservation Commission (COGCC) releases production and location data for all gas wells in their jurisdiction [1]. We grouped wells within 50 meters of a given well into sites to establish a distribution of the number of wells per site following the method described by Omara et al. [2]. We then assumed that there were, on average, 650 components per well at each site. The estimate includes all components at the site, rather than the components that are part of the wellheads and is similar to the natural gas well site model plant developed by the EPA [3]. Every 
Non - peer reviewed pre - print submitted to EarthArXiv

Monte Carlo iteration of the model used in this work contained 100 sites, but the number of wells at each site was chosen randomly for each iteration.

\section{S2.2 Fugitive emissions and unclassified vents}

Our simulation of fugitive emissions and unclassified vents is driven by publicly available data. While numerous studies have measured emissions at the component level on well pads, few have distinguished between vents and fugitive emissions. Here, we define vents as any emissions that occur by design and will not be affected by an LDAR program. We define fugitive emissions as emissions that are unintentional and can be stopped if detected.

While some emissions are simple to classify on site, others are prohibitively complex for typical survey teams. For example, a pressure relief valve may be emitting when surveyors are on site but determining whether that emission is a vent caused by a temporary high-pressure condition, a leak caused by a faulty valve, or a leak caused by a faulty piece of equipment upstream of the valve may be beyond the scope of the detection survey. Furthermore, not all operators and surveyors use the same definition of a fugitive emission. For example, some jurisdictions classify all tank related emissions as vents [4] while others distinguish between different types of tank emissions [5].

Therefore, we designate $45 \%$ of emissions as vents. In practice, field students at oil and gas facilities have found a wide variation in the fraction of emissions that can be classified as vents. The effect of changing this percentage is examined in the sensitivity analysis in section S4.

\section{S2.2.1 Emission rate distribution}

We compiled a database of component-level emission surveys based on publicly available data to populate the emissions model in FEAST. To be included in the database, the surveys were required to meet the following three criteria:

1. The surveys were conducted at upstream production facilities,

2. The study included all emissions that could be measured at production facilities,

3. Emission rates were measured at the component level. Criteria two excludes studies that focused on a particular component type. This restriction allowed direct use of the emissions dataset to generate a distribution of fugitive emission rates for well sites. Future work may use component-specific emission distributions and require component-type activity data, but supporting that complexity is beyond the scope of this analysis. Criteria two ensures that the emission data used are from the industry segment of interest to this work. Criteria three is necessary because detection technologies that identify emissions at the component level were modeled in this work. Furthermore, we can aggregate component-level data to the equipment-level, while the reverse disaggregation is typically impossible without information on emitting components.

Five major studies were identified that satisfy all three criteria as summarized in . Comparing the studies in pairs using a two sample Kolmogorov-Smirnov test shows that each study resulted in an independent emission distribution (all pair-wise p-values $<<0.01$ ). Several factors contribute to the differences in the observed distributions. First, the studies took place in disparate geologic basins, operating environments, and geographic locations. The five surveys spanned sites in Alberta, California, Colorado, Arkansas, Texas, and Appalachia. The 
117 characteristics of gas fields across these locations are very different. For example, most wells in 118 California are vertical with one well per pad, while most wells in Colorado are drilled 119 horizontally and well pads service multiple wells. Gas wells in Appalachia tend to produce dry gas while the fields in Alberta produce gas and oil. The variety of drilling practices, resource characteristics and infrastructure are expected to affect the size distribution of emissions.

Furthermore, the studies used distinct methods to identify emissions. Allen and Ravikumar used optical gas imaging (OGI) technology to identify emissions. ERG identified some emissions using an OGI camera and others with a Toxic Vapor Analyzer (TVA). The emission distributions associated with each technology are shown separately in Figure S1. Bell used an OGI camera and a Tunable Diode Laser Absorption Spectroscopy (TDLAS) technology to detect emissions. Emissions from both methods are combined in one distribution because the emissions were not segregated by detection method in the study. Kuo also used a TDLAS technology for detection.

Table S1 Studies included in populating the empirical emission-size distributions in FEAST. Year indicates the year when the study was published (not the year when measurements were made). The number of sites refers to the number of well pad production sites included in the study. The number of emissions identified includes both measured and unmeasured (non-quantified, but only detected)

135 emissions.

\begin{tabular}{|l|l|l|l|l|l|l|}
\hline Citation & Lead author & Year & $\begin{array}{l}\text { Number of } \\
\text { sites }\end{array}$ & $\begin{array}{l}\text { Number } \\
\text { of wells }\end{array}$ & $\begin{array}{l}\text { Number of } \\
\text { emissions identified }\end{array}$ & $\begin{array}{l}\text { Survey } \\
\text { method }\end{array}$ \\
\hline$[6]$ & Allen & 2013 & 150 & 489 & 769 & OGI \\
\hline$[7]$ & ERG & 2011 & 375 & 1121 & 1193 & OGI \\
\hline$[7]$ & ERG & 2011 & & 112 & 756 & TVA \\
\hline$[8]$ & Kuo & 2012 & & 128 & 94 & TDLAS \\
\hline$[9]$ & Bell & 2017 & 261 & & 322 & $\begin{array}{l}\text { OGI and } \\
\text { TDLAS }\end{array}$ \\
\hline$[4]$ & Ravikumar & 2020 & 27 & & 1236 & OGI \\
\hline
\end{tabular}




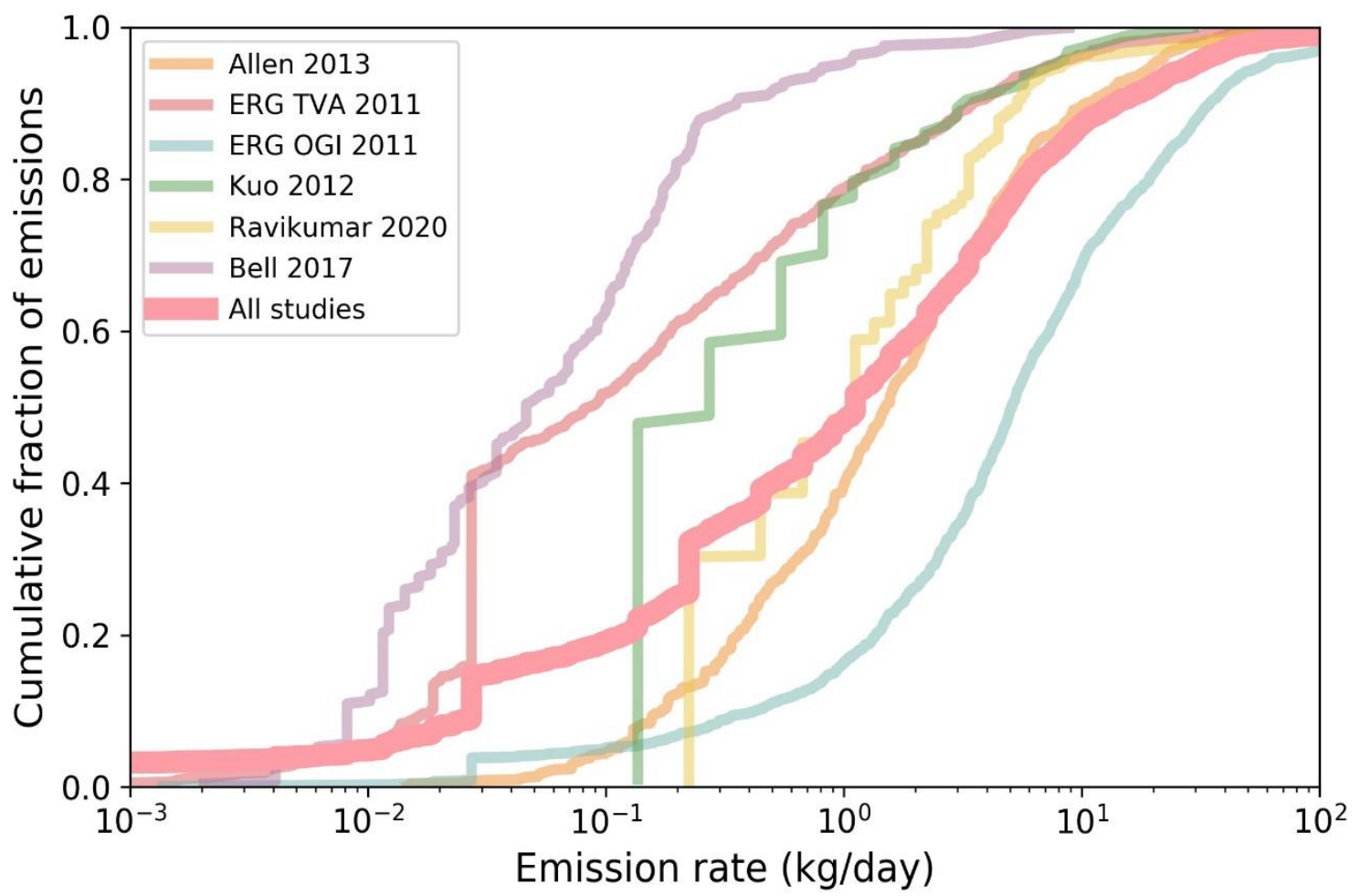

Figure S1 Component-level emission-size distribution measured in each of the studies summarized in Table S1.

\section{S2.3 Pneumatic controllers}

Pneumatic controllers have been estimated to contribute $23 \%$ and $27 \%$ of total natural gas emissions from the production sector [10], [11]. Since all studies in the emissions distribution database except Bell 2017 include pneumatic controller emissions, pneumatic controllers are treated as an unclassified vent in this work. As a result, the frequent short duration characteristics of pneumatic emissions are not captured by this model.

\section{S2.3.1 Emission distribution sensitivity}

The component-level emissions distribution has at least three sources of uncertainty:

1. Finite sample size

2. Potential for bias in measurements

3. Regional variability

The heavy tailed distribution of emission sizes and associated uncertainty has been broadly acknowledged [12]. The bootstrapping method used in this work to select emission sizes avoids biasing the data with a parametric model. However, the method is limited by the finite sample size and does not simulate any emissions larger than those captured in the empirical data.

There is also a bias introduced to our work because only emissions that were detected and measured can be included. In some cases, safety or access related challenges may have prevented important emissions from being quantified in field campaigns. For example, Ravikumar et al. reported that all tank emissions detected in their study were not directly quantified. Previous studies have shown that tank emissions are often a source of large emissions that account for 
over one third of total emissions from production sites [4]. To overcome this bias, Ravikumar et al. appended the emission data set with an estimate of tank emissions based on results from other studies. By incorporating many component level emission studies using a variety of detection methods, we aim to reduce the potential for systematic bias in the emission dataset. As more component-level studies are made publicly available and incorporated in this model, the empirical size distributions will become more representative of actual size distributions.

We explore the sensitivity of results to the emission rate distribution in the sensitivity analysis. In addition to choosing emission rates from the empirical distribution using bootstrapping, we also use a constrained power law distribution, as described below. The power law distribution allows us to observe the impact of manipulating the large emission tail of the distribution.

\section{S2.3.2 Bootstrap emission size selection}

The skew of the emission size distribution is well documented and influences the efficacy of leak detection programs [12]. Bootstrap sampling of emission rates from an empirical distribution is the most robust method for representing heavy-tailed emission distributions [12]. In this work, we used bootstrap sampling to generate results for Figures 1-3 in the main text and employed a constrained power-law distribution in sensitivity analysis. Specifically, every emission generated by FEAST is assigned an emission rate drawn randomly with replacement from the emission rate database.

\section{S2.3.3 Power law emission size selection}

Power law emission distributions are useful for exploring the impact of emission distribution on mitigation equivalence. The results shown in Figure 4 in the main text use power law emission distributions. We generated the distributions using bootstrap sampling from the compiled emission database up to the $80^{\text {th }}$ percentile and a power law distribution of emission rates for larger emissions. The probability density function for emission rates greater than the $80^{\text {th }}$ percentile is shown in Equation $\mathrm{S} 1$, where $\alpha$ is a tuning parameter and $x$ is an emission rate. In order to prevent impossible emission rates, the power law distribution was not allowed to exceed the simulated gas production rate at the site where the emission occurred $\left(Q_{\text {site }}\right)$. $Q_{\text {site }}$ was drawn from the distribution of production rates at sites in Colorado with the same number of wells as the simulated site [1].

$$
p\left(x \mid x>x_{80 \%}\right)=\min \left(\frac{\alpha-1}{x_{80 \%}}\left(\frac{x}{x_{80 \%}}\right)^{-\alpha}, Q_{\text {site }}\right)
$$

The method described above allows direct manipulation of the tail of the empirical distribution - to which tiered detection methods are particularly sensitive, and data are sparse - while maintaining the median emission rate. In contrast, the tail of a lognormal distribution cannot be tuned without also affecting the median emission rate and lognormal distributions have been shown to under-estimate the tail of component-level emission distributions [12].

The expected number of emissions present during the undirected inspection and maintenance (UDIM) scenario is determined based on the average emissions per well in the compiled emission data set, equivalent to about 1.5 emissions per well. The number of components leaking at the beginning of the simulation is determined by drawing from a binomial distribution with a 
leak probability that results in an average of 1.5 emissions per well. The mean duration of emissions in the UDIM scenario $\left(T_{E m i t}\right)$ is set according to Equation ( $\mathrm{S} 2$, where $N_{e}$ is the expected number of emissions and $N_{c}$ is the number of components. In an UDIM simulation, the duration of every fugitive emission and unclassified vent is drawn from an exponential distribution parameterized with $T_{E m i t}$. Setting $T_{E m i t}$ according to Equation (S2 ensures that while the number of emissions varies during and between simulations, the expectation value of the number of emissions is consistent with emission rates observed in the field.

$$
T_{\text {Emit }}=\frac{N_{e}}{N_{c}} T_{\text {Failure }}
$$

\section{S2.4 Unloading events}

Unloading emissions refer to gas that is vented in the process of removing accumulated liquids from wells. While some wells require automated plunger lift systems and unload hundreds of times per year, other wells only require a few unloading events per year and can be triggered manually. The frequency of unloading events varies between geologic basins. This work simulates production sites in the DJ basin, where unloading events are relatively rare.

Unloading emissions are unique because they are not included in the component-level surveys used for the fugitive and uncategorized vent models. They are modeled explicitly in FEAST as it can be a significant contributor to total methane emissions in liquids-rich basins. The unloading model allows for three types of wells: wells that do not unload, wells that use plungers to remove liquids, and wells that unload without a plunger. The parameters used to specify these emissions are provided in Table S2. The number of events per year and the emissions per event are both set to average values calculated using emission data from the EPA greenhouse gas reporting program (GHGRP) for the DJ basin [13]. The duration of the events is set to the average value of the duration distribution reported by Zaimes et al [14].

Table S2 Unloading parameters in FEAST for wells with and without plunger lifts.

\begin{tabular}{|l|l|l|}
\hline & Plunger unloading & No plunger unloading \\
\hline Average duration (minutes) & 34 & 83 \\
\hline Average emission rate $(\mathrm{kg} / \mathrm{hr})$ & 181 & 277 \\
\hline Frequency (\#/year) & 6 & 0.1 \\
\hline
\end{tabular}

\section{S2.4.1 Number of unloading wells}

All facilities that emit more than 25,000 metric tons $\mathrm{CO}_{2} \mathrm{e}$ greenhouse gases annually are required to report to the GHGRP. In the case of onshore oil and gas production, a "facility" is defined as all wells and associated production equipment in a geologic basin owned by a single entity [15]. In 2017, 16 companies operating 23,037 wells in Colorado reported emissions.

The fraction of wells that reported plunger and no-plunger unloading events were used to designate the number of unloading wells in the base case simulations. Specifically, the GHGRP reported $2662(11.6 \%)$ plunger-unloading wells and $541(2.3 \%)$ no-plunger-unloading wells for the state of Colorado. 


\section{S2.4.2 Emission profiles}

To simulate LDAR programs, FEAST requires a duration and emission rate for every emission. facility for plunger and non-plunger wells separately. Therefore, the GHGRP data can be used to calculate the average emission mass per event for plunger and non-plunger wells. The GHGRP does not provide unloading emission data at the well level or event level, so a distribution of emission sizes cannot be determined. The GHGRP does not provide the duration of events either, so a flux rate cannot be directly estimated from the GHGRP data. Instead, the average emission duration reported by Zaimes is used for all unloading events in the base case simulation, and the flux rate is calculated based on the average emission volume and duration. Zaimes reports that 3 out of 2532 venting wells use automatic unloading systems in the DJ basin. Therefore, the manual no-plunger (4974 seconds) and manual plunger (2059 seconds) durations are used.

\section{S2.4.3 Unloading model uncertainties}

All facilities in Colorado in 2017 estimated emissions using Method 2 or Method 3 specified in 40 CFR Subpart W $\S 98.233$ based on the type of unloading (plunger or no-plunger), well geometry and event duration [16]. In 2014, Allen et al. showed that the equations used by Method 2 were not significantly correlated with measurements made at 32 non-plunger lift wells, although the mean estimate was statistically similar to the mean measured emission. Conversely, measurements at 75 wells with plunger lifts were found to be significantly correlated with the Method 3 estimates $\left(r^{2}=0.08, p=0.015\right)$, but the mean estimate was $44 \%$ lower than the mean measured emission. Allen's data illustrates that the accuracy of the estimates provided to the GHGRP is limited even if the parameters required by the method are well known.

Zaimes et al recently showed that the GHGRP data is too limited to quantitatively represent the range of emissions associated with unloading events [14]. To improve on the GHGRP data for Monte Carlo modeling, Zaimes combined data from three sources - DIDesktop, Allen et al. unloading measurement campaign, and the GHGRP. Zaimes' method relies on the GHGRP Method 2 and 3 correlation equations to estimate unloading emissions based on well characteristics and does not account for the systematic errors in those methods that Allen's measurements suggest. However, Zaimes' approach enables Monte Carlo modeling of the uncertainty in emissions while the GHGRP data does not provide for the distribution of well parameters that result in the reported emission estimates.

Despite these limitations, the base-case simulations presented here use GHGRP data to represent the current emission estimate available to regulators. The presence of unloading events can affect simulation results by triggering site-level survey programs to dispatch ground crews. However, the frequency of unloading events in this simulation - an average of one 34-minute event every 63 days at wells with plungers - results in a low probability of an unloading event affecting an LDAR program. Users of the FEAST model attempting to evaluate LDAR programs in basins with significant unloading events should considering incorporating basin-specific data on unloading emisisons to improve the accuracy of results. 


\section{S2.5 Leak production rate and UDIM repair rate}

The leak production rate is a primary driver of uncertainty, as shown in the sensitivity analysis. The base case leak production rate of 3 leaks per site per year used in this work is within the range of previously published estimates and empirically supported by a new estimate based on the regulated OGI survey reports released by the Colorado Department of Public Health and Environment (CDPHE).

The CDPHE requires OGI surveys at all production sites monthly, quarterly, semi-annually, or discovered at each survey frequency by the time between surveys, then computed the average across all survey frequencies weighted by the number of sites. The resulting average leak production rate estimate in this case was 2 leaks per site per year.

Unchecked, the leak production rate estimated above results in unrealistic growth in emissions over timescales longer than a year. In keeping with prior publications, we assume that an undirected maintenance process results in a steady state number of emissions equal to that observed in the empirical emission data set. We explore sensitivity of the results to both the UDIM repair rate and the leak production rate in Section S4.

\section{S3. LDAR programs}

LDAR program simulations require specifying three classes of variables:

1. Detection technology characteristics

2. Program implementation protocols

3. Repair protocols

Each of these classes of variables are described below.

\section{S3.1.1 Detection technology characteristics}

Detection technologies in FEAST are defined by their probability of detecting emissions and their ability to disaggregate overlapping emissions. Depending on the technology, the probability of detection may depend on flux, wind speed, operator experience, and other exogenous parameters. Prior modeling efforts used a Gaussian plume dispersion model, empirical wind speed distributions, and a range of atmospheric stability classes to determine the concentration of emitted gases in the volume surrounding an emission source. The concentration profile was then used to calculate the signal in a variety of detection technologies [17]. More recently, empirical, one dimensional probability of detection curves based on emission rate were favored over plume modeling [18]. The empirical approach is powerful because it uses measured technology performance directly. However, to effectively model field performance of new technologies, the probability of detection curve must represent the range of conditions that will be realized in the field. This will result in a much broader probability of detection curve than can be measured at a test site under a single set of conditions. The effect of meteorology, user experience and other variables on probability of detection will need to be accounted for in order to extrapolate from test conditions to field applications [19]-[21].

When the data are available, detection technologies should be modelled using probability of detection surfaces that capture the variables most likely to impact detection for each technology. However, adding variables to technology models will introduce the "curse of dimensionality" to 
testing requirements. As suggested by Barchyn et al. [19], requiring that detection technologies are only applied under a particular range of conditions would limit the need for testing. Future versions of FEAST will support probability of detection surfaces with two independent variables as well as operating envelopes.

In this work, the probability of detection for a given flux is calculated according to Equation (S3), where $f$ is the emission flux, $\mu$ is the log of the median detection threshold - the emission rate with a $50 \%$ probability of detection - and $\lambda$ is a fitting parameter that defines the slope of the curve. The median detection threshold is taken from recently published controlled release tests of methane detection technologies [18], [20], [22]

$$
p(d \mid f)=0.5+0.5 * \operatorname{erf}\left(\frac{\ln (f)-\mu}{\sqrt{2} \lambda}\right)
$$

Figure S2 shows the PoD curves used in simulations presented in Figures 1-2 in the main text.

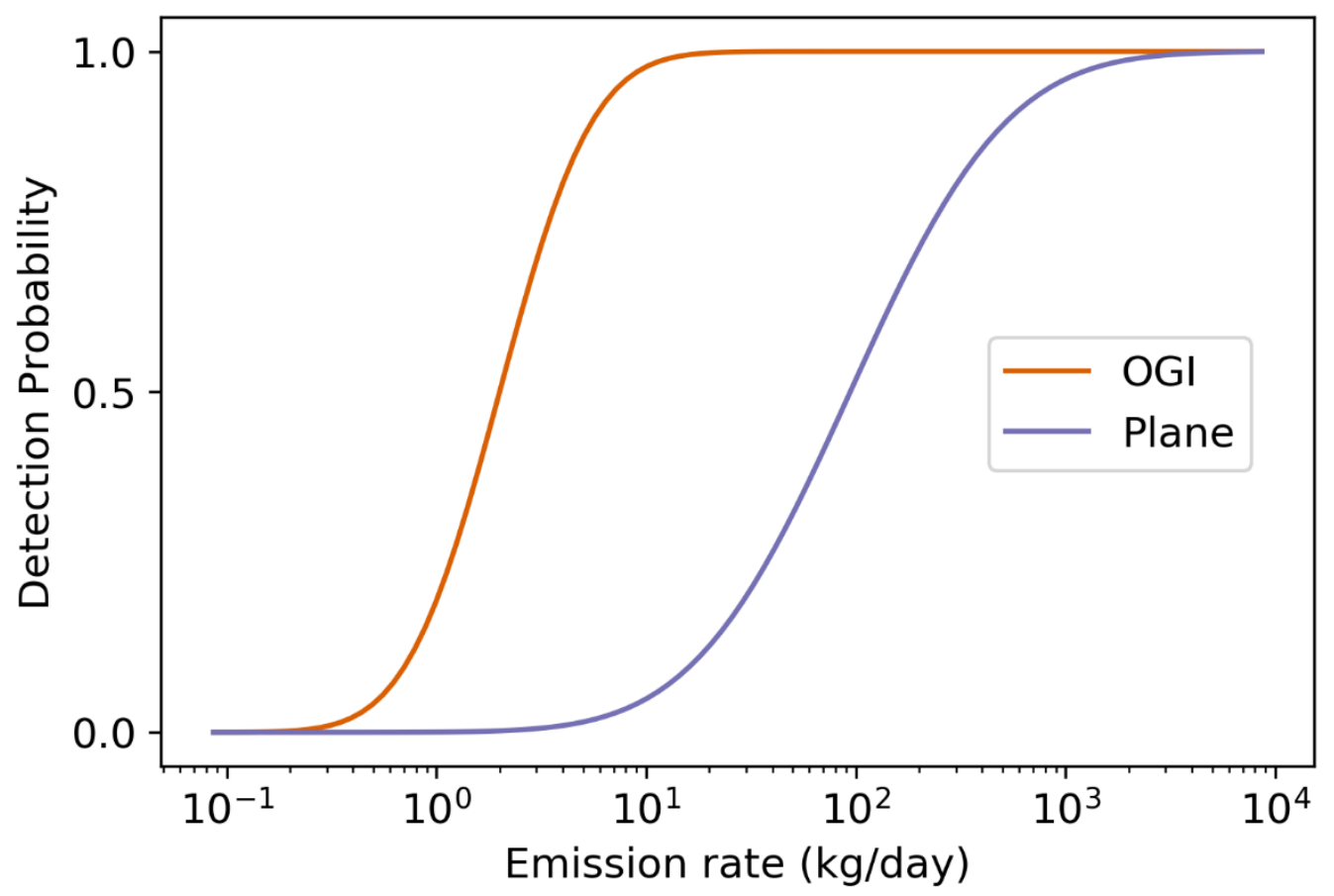

Figure S2 Probability of detection curves for the OGI (orange) and the plane-based (purple) technology modeled in this study.

\section{S3.1.2 Program implementation protocols}

LDAR program implementation protocols specify how a detection technology will be used.

These protocols include the survey frequency, and relationships between detection methods. For the Plane + OGI programs represented here, the LDAR program specifies that an OGI detection method should be dispatched at any site where the plane detects emissions. LDAR protocols also specify which repair methods should be dispatched if an OGI detection method identifies a repairable emission. 
S3.1.3 Repair protocols

Repair methods cause an emission to cease and are characterized by the time between when they are called and when the repair occurs. Component level surveys are assigned a 1 day delay between detection and repair, while site level surveys are assigned a 1 week delay.

\section{S3.2 Calculating mitigation costs}

FEAST calculates costs based on the survey speed (components/hour) and hourly cost in the case of component-level surveys. Costs for site level methods are calculated based on a cost per site parameter. Repair costs are also assigned to leaks repaired by either UDIM or an LDAR program. The total cost of an LDAR program includes survey costs and the difference in repair costs between the LDAR scenario and the UDIM only scenario. Since most leaks are repaired by UDIM over the course of the three-year simulation, the net repair costs assigned to LDAR programs is small compared to the costs of survey.

Mitigation cost is calculated as the ratio of total cost to avoided greenhouse gas emissions, as shown in Equation (S4). GWP $\mathrm{CH}_{4}$ is the global warming potential of methane, set to 34 in this case following estimates from the IPCC $5^{\text {th }}$ Assessment Report [23].

$$
\text { Mitigation cost }=\frac{\text { Total cost }}{\text { Methane emissions mitigated (metric ton }) \times G W P_{\mathrm{CH}_{4}}}
$$

The results of an equivalence analysis are sensitive to the parameters specified in FEAST simulations. In the main text, we highlighted the sensitivity of results to survey frequency in Figure 2, detector sensitivity and survey frequency in Figure 3, and emission distribution skew and detector sensitivity in Figure 4. These figures show that the model is neither linear nor additive: local derivatives of results with respect to one input variable are not indicative of model behavior across the range of possible input values, and the impact on results of changing two input values cannot necessarily be approximated by adding the impact of changing each input value independently. The "one-at-time" style sensitivity analysis presented in previous FEAST publications provides insight into the sensitivity of the model to input parameters near the "base case" scenario but is difficult to use in assessing the overall sensitivity of the model. Allowing multiple input parameters to vary simultaneously provides better representation of the sensitivity of the model to changes in the underlying assumptions [24].

Our sensitivity analysis is designed to assess the confidence that a regulator could have in an equivalence assessment given the existing data to support global parameters in FEAST. Global parameters refer to parameters that are consistent across all Monte Carlo iterations of a scenario, such as the choice of leak production rate, emission rate distribution, and vent fraction. Specifically, we ask: given realistic uncertainties in global parameters defined for FEAST simulations, how confident can a regulator be that two distinct LDAR programs that appear to be equivalent under the assumptions described for this work would achieve equivalent results in the 
field? To answer this question, we consider a Plane + OGI program with a survey frequency of 9/year, and an OGI program with a survey frequency of 2/year.

Figure 2 in the main text shows that the mean emission rates are equal for these LDAR programs but there is variability in those emission rates due to several random process included in FEAST. The random processes in FEAST include the Poisson process representing emission creation, the distribution of fugitive emission sizes, and the PoD surface. These processes are inherently probabilistic: with perfect information, a regulator would still expect to see variability in results.

To these random processes, we now add a distribution for parameters in FEAST that may not be inherently probabilistic but are poorly constrained by available empirical data. We suppose that the LDAR program parameters are known precisely, but the leak production rate, UDIM repair rate, emission distribution, and vent fraction are only constrained by existing empirical data. The range of results gives a quantitative illustration of the confidence that a regulator could have in an equivalence assessment given perfect information about an LDAR program and illustrates the areas where additional empirical data would improve confidence the most.

\section{S4.1 Emission rate distribution}

Figure 4 showed that the emission rate distribution affects the level of mitigation that LDAR programs can achieve. How confident can a user be in the emission distribution used by FEAST?

We examine the sensitivity to the component level distribution by invoking different empirical component-level leak distributions. In each iteration of the sensitivity analysis, we randomly select results from one of the five studies listed in

. The study used in each iteration is treated as a categorical variable, with equal probability assigned to each study.

\section{S4.2 Leak production rate and UDIM repair rate}

The leak production rate $\left(R_{f}-\right.$ failures per component per day) and UDIM repair rate $\left(R_{R}-\right.$ repairs per leak per day) are related to each other in FEAST by Equation (S5, where $N_{c}(t)$ and $N_{L}(t)$ are the number of components and number of leaks as functions of time $(t)$. The \langle\rangle notation indicates an expectation value (i.e., the theoretical average across an infinite number of iterations and timesteps).

$$
\left\langle\frac{d N_{L}(t)}{d t}\right\rangle=N_{c} R_{f}-N_{L}(t) R_{R}
$$

Setting a boundary condition of $N_{L}(t=0)=N_{L 0}$, we can solve Equation (S5 to derive an expression for $N_{L}(t)$ as shown in Equation (S6.

$$
\left\langle N_{L}(t)\right\rangle=N_{L 0}+N_{c} \frac{R_{f}}{R_{R}}\left(1-\exp \left(-R_{R} t\right)\right)
$$

$R_{f}$ and $R_{R}$ could be determined by fitting Equation (S6 to the time evolution of emission counts at production sites (for example, by conducting repeated surveys of the same sites without reporting emission to operators). However, such data are not presently available. Therefore, we developed a range of likely values for $R_{f}$ and $R_{R}$ in this sensitivity analysis. 

production equipment in Colorado. First, we consider the case in which the UDIM repairs are rare between surveys $\left(R_{R} t_{s} \ll 1\right)$ where $t_{s}$ is the time between surveys. We also assume that the vast majority of leaks are detected and repaired at each survey $\left.\left(\left\langle N_{L}\left(t_{S}\right)\right\rangle\right\rangle N_{L 0}\right)$. In that case a Taylor expansion of Equation (S6) reveals that $\left\langle N_{L}\left(t_{s}\right)\right\rangle \approx N_{c} R_{f} t_{s}$. Therefore, the leak production rate $R_{f}$ can be estimated based on the number of leaks detected in periodic surveys if the survey interval and number of components surveyed is known.

In order to develop a distribution for $R_{f}$ to use in sensitivity analysis, we consider surveys conducted at different site types and frequencies in Colorado. Specifically, we consider sites surveyed once, twice, four and twelve times per year. The survey frequency required by the state of Colorado depends on the site characteristics (larger sites generally require more frequent surveys) and site location (sites in non-attainment zones require more frequent surveys) [5]. The data are summarized in Table S3. In the sensitivity analysis, each survey frequency is selected with a probability equal to the fraction of total sites included in the reports. In comparison, the leak production rate used for Figures 1-4 in the main text is 3 leaks per site per year, [25] used 2.4 leaks per well per year, and Fox et al. reported a mean leak production rate estimate of 9.5 leaks per site per year [26]. To convert the reported leaks per site to a component-level leak production rate, we divide by 1235 components per site (assumes 650 components per well and an average of 1.9 wells per site).

\section{Table S3 Summary of data reported in LDAR surveys regulated by CDPHE}

\begin{tabular}{|l|l|l|l|}
\hline $\begin{array}{l}\text { Survey frequency } \\
\text { (surveys per year) }\end{array}$ & $\begin{array}{l}\text { Leaks identified per } \\
\text { site per year }\end{array}$ & $\begin{array}{l}\text { Inspections that } \\
\text { occurred }\end{array}$ & $\begin{array}{l}\text { Approximate number } \\
\text { of sites based on } \\
\text { number of } \\
\text { inspections }\end{array}$ \\
\hline 1 & 0.8 & 4246 & 4246 \\
\hline 2 & 0.7 & 4907 & 4907 \\
\hline 3 & 4.2 & 3766 & 942 \\
\hline 4 & 12.8 & 10914 & 910 \\
\hline
\end{tabular}

\section{9}

460

461

462

463

464

465

466

467

468

469

470

471

472

The leak production rate estimated by long survey frequencies of six months or one year in Table S3 likely underestimate the leak production rate to due to UDIM repairs that occur between surveys. Conversely, the leak production rate in monthly surveys is likely overestimated due to the regulator requiring monthly surveys only at sites expected to have high emissions. Therefore, the range indicated by Table S3 may exaggerate the range of likely component-level leak production rates.

\section{S4.3 UDIM repair rate distribution}

In Figures 1-4 of the main text, the UDIM repair rate was set to guarantee that in the absence of an LDAR program, the frequency of emissions in the simulation tended toward the frequency of emissions observed in the included field studies. Here, we choose the UDIM repair rate from a triangular distribution. The inverse of the UDIM repair rate provides the mean duration of an 
emission under UDIM. The mode of the triangular distribution is set to a mean duration of 208 days, the same value used in simulations for the main text. The minimum UDIM emission duration is set to 100 days, and the maximum is set to three years.

\section{S4.4 Vent fraction}

In the main text, the vent fraction excluding unloading events was set to $46 \%$. Unloading events were rare in these simulations, increasing the overall vent fraction to $47 \%$ despite their large emission rate. Prior studies have identified vented emissions contributing between 55\% and 90\% of total emissions (with the highest vent fractions coming from compressor stations rather than well sites) [4], [27]-[29]. In this sensitivity analysis, we choose the vent fraction from a uniform distribution ranging from 30 to $60 \%$. The high end of the vent fraction range is consistent with emission fractions observed at well sites, and the low end of the range allows for the possibility that some emissions classified as vents (such as fugitive tank emissions) would be mitigated in a practical LDAR program.

\section{S4.5 Confidence in equivalence assessments}

We define an equivalence metric $\phi$ in Equation (S7) to quantify the equivalence between two proposed LDAR programs. $F$ denotes the total fugitive emissions under an LDAR program. The subscript plane indicates the Plane + OGI LDAR program with a survey frequency of 9/year and median detection threshold of $94 \mathrm{~kg} /$ day, while the subscript $O G I$ refers to the component level survey with a frequency of 2/year and a detection threshold of $2 \mathrm{~kg} / \mathrm{day}$.

$$
\phi=\frac{F_{\text {plane }}-F_{O G I}}{F_{\text {plane }}+F_{O G I}}
$$

Values of $\phi<0$ imply thate the Plane + OGI program reduces emissions more than the OGI program while $\phi>0$ implies that the OGI program outperforms the Plane + OGI program. $\phi$ is bounded between -1 and 1 . When the two programs achieve equal mitigation, $\phi=0$.

Figure S3 shows a histogram of $\phi$ resulting from 10,000 iterations of FEAST with values of the leak production rate, emission distribution, UDIM repair rate and vent fraction drawn from the distributions described above. $12 \%$ of the iterations result in the Plane + OGI program achieving equivalent mitigation to the OGI program. This implies that, although the assumptions presented in the main text of the paper result in the Plane + OGI program achieving equal mitigation to the OGI program at these survey frequencies, we cannot be confident in that result given existing uncertainty in the leak production rate and emission distribution. The following section investigates the type of empirical data that would be most valuable to increasing confidence in equivalence evaluations. 


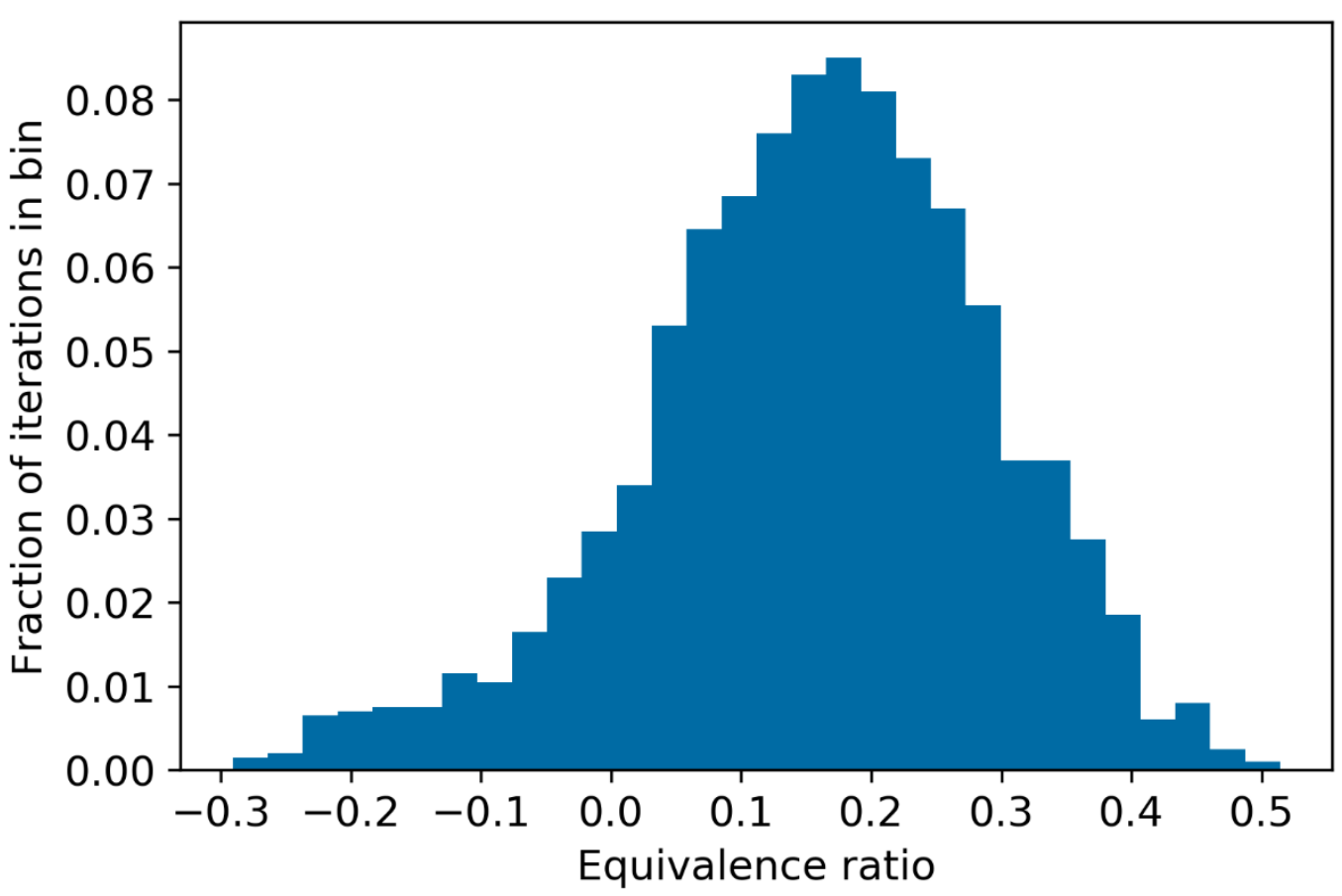

Figure S3 Distribution of the equivalence ratio while varying input parameters to FEAST.

\section{S4.6 Sensitivity of results to input parameters}

512 The following figures illustrate the sensitivity of the model to the four parameters considered in this section. We find the greatest value will come from reducing uncertainty in the leak production rate, followed by the emission distribution. The results have little sensitivity to the vent fraction.

Figure S4 shows the distribution of the equivalence metric under different leak production rates. is significant overlap between the distributions: other sources of uncertainty are also important. 
Non - peer reviewed pre - print submitted to EarthArXiv

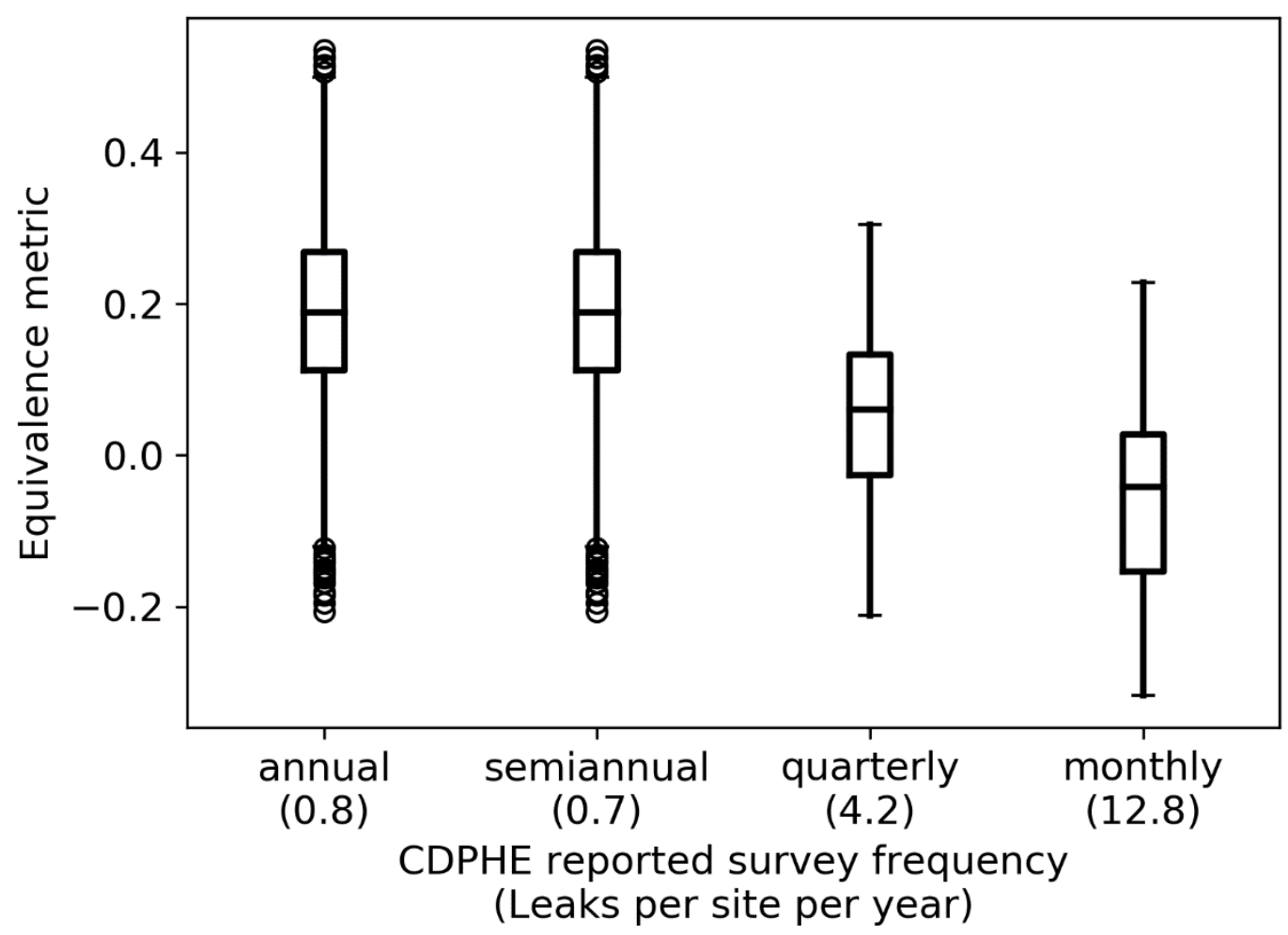

Figure S4 Distribution of the equivalence ratio when the leak production rate is estimated using results from OGI surveys regulated by the Colorado Department of Public Health and Environment (CDPHE).

524 Different survey frequencies are applied to different production sites, resulting in distinct leaks per site per 525 year estimates when the data are separated by survey frequency.

526 Figure S5 shows the distribution of the equivalence metric under different emission distributions. 527 The data show that the results are sensitive to the emission distribution chosen for the 528 simulations. To develop fair equivalence metrics, regulators must choose an emission 529 distribution that accurately represents their facilities. 


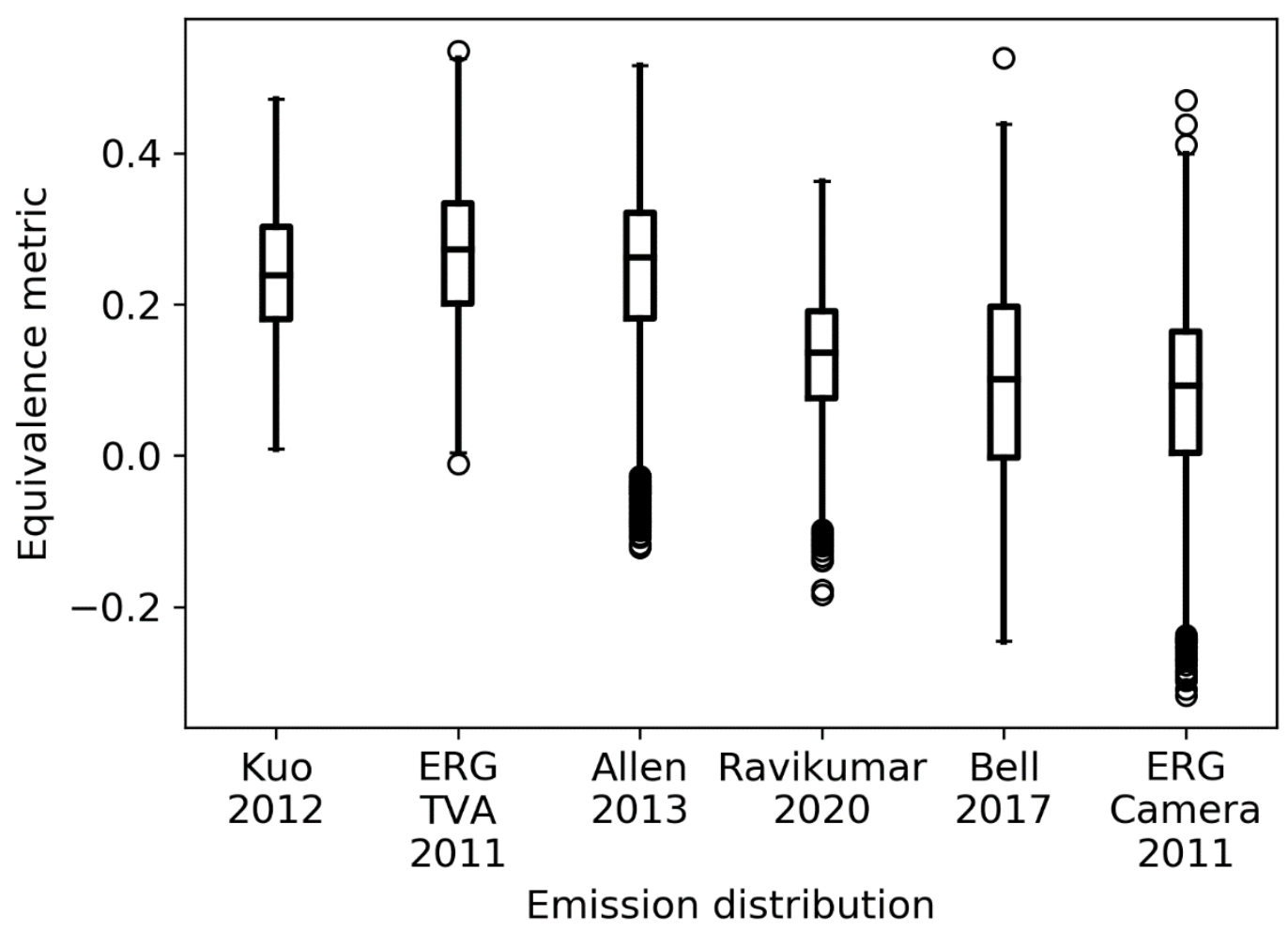

Figure S5 Distribution of the equivalence metric under different emission distributions. Emission distributions are in order of increasing mean emission rate.

Figure S6 shows results from all monte carlo iterations plotted against the UDIM leak lifetime chosen for each iteration (1/UDIM repair rate). We see that the variance is dominated by other sources of uncertainty, but there is a correlation between UDIM leak lifetime and Equivalence metric (r-value of 0.082 and p-value <0.001). The impact of the UDIM leak lifetime is limited because most emissions are repaired by the LDAR program in both the OGI and the Plane + OGI cases rather than by the UDIM process.

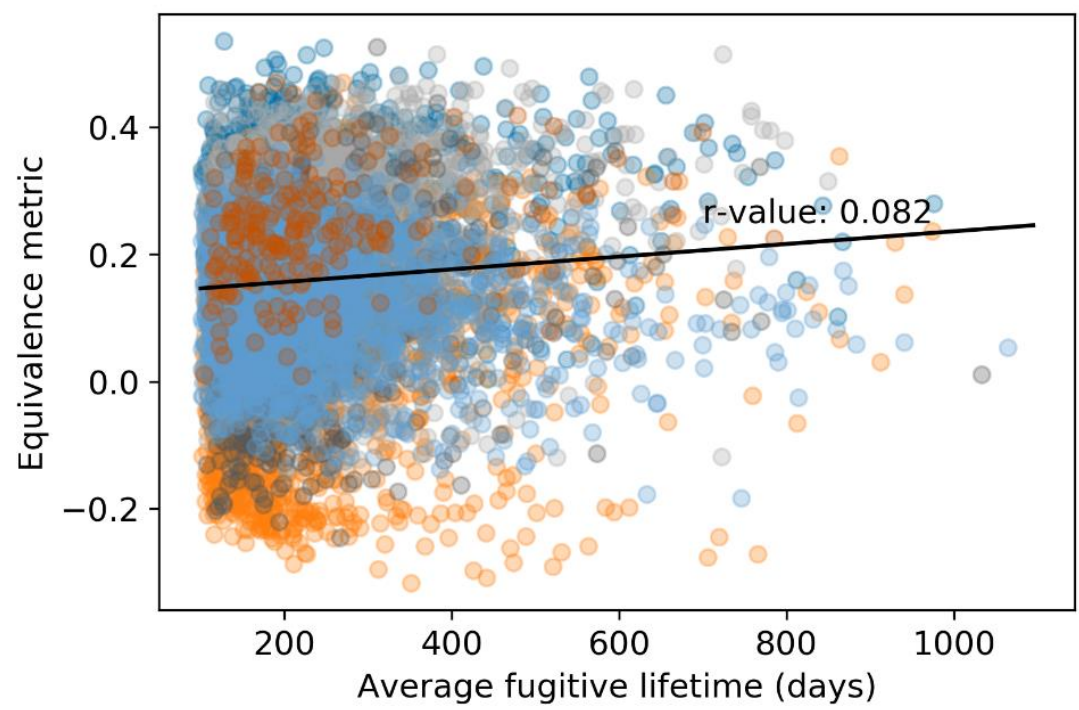

\begin{tabular}{|l|}
\hline ERG TVA 2011 \\
ERG Camera 2011 \\
Allen 2013 \\
Bell 2017 \\
Ravikumar 2020 \\
Kuo 2012
\end{tabular}

Figure S6 Equivalence metric of all iterations plotted against the expected leak lifetime under UDIM. The best linear fit to the data is plotted along with the correlation coefficient of the fit. 
542 Finally, Figure S7 shows results from all Monte Carlo iterations plotted against the vent fraction. 543 The data suggest that equivalence, in this case, is weakly dependent on the vent fraction in the 544 range tested (p-value of 0.1). This result is due to the Plane + OGI flagging the same sites for 545 follow up surveys regardless of whether emissions are classified as fugitive or vent. Increasing 546 the vent fraction decreases the emission mitigation from those flagged sites equally for both 547 types of LDAR program.

To illustrate the impact of vent fraction on the equivalence ratio, consider the example in Table S4. There are two sites in the example. In every case, there are two emissions at Site $1(0.2$ and $1.0 \mathrm{~g} / \mathrm{s})$ and three emissions at Site $2(0.2,0.1$ and $0.01 \mathrm{~g} / \mathrm{s})$. The emissions are classified as vents or leaks in four distinct scenarios. In case 1, all of the emissions are classified as fugitive emissions. In cases 2-4, two of the emissions are classified as vents. We imagine a tiered LDAR program that flags all sites with emissions $>1.0 \mathrm{~g} / \mathrm{s}$ for follow up action. We also consider a component level inspection program that repairs all fugitive emissions $>0.05 \mathrm{~g} / \mathrm{s}$. In short, the tiered program repairs all fugitive emissions at Site 1, and the component level program repairs all fugitive emissions except the $0.01 \mathrm{~g} / \mathrm{s}$ emission.

Table S4 shows that the effect of the vent fraction on the equivalence ratio depends on which emissions are classified as vents. Case 1 has a vent fraction of 0 resulting in an equivalence ratio of 0.94. Cases 2-4 have higher vent fractions, but the equivalence ratio can be greater than, less than or equal to the equivalence ratio of Case 1 . The effect of the vent fraction on the equivalence ratio depends on the relative sizes of the emissions classified as vents and fugitive emissions.

Table S4 Example of the impact of vent fraction on equivalence ratio

\begin{tabular}{|l|l|l|l|l|l|l|l|}
\hline & \multicolumn{2}{|c|}{ Site 1 emissions $(\mathrm{g} / \mathrm{s})$} & \multicolumn{2}{l|}{ Site 2 emissions $(\mathrm{g} / \mathrm{s})$} & $\begin{array}{l}\text { Number } \\
\text { of vents }\end{array}$ & $\begin{array}{l}\text { Vent } \\
\text { fraction }\end{array}$ & $\begin{array}{l}\text { Equivalence } \\
\text { metric }\end{array}$ \\
\hline & Fugitive & Vent & Fugitive & Vent & 0.94 \\
\hline Case 1 & $0.2,1.0$ & - & $0.2,0.1,0.01$ & - & 0 & 0 & 0.94 \\
\hline Case 2 & 1.0 & 0.2 & $0.1,0.01$ & 0.2 & 2 & $26 \%$ & 0.83 \\
\hline Case 3 & - & $0.2,1.0$ & $0.2,0.1,0.01$ & - & 2 & $79 \%$ & 0.94 \\
\hline Case 4 & $0.2,1.0$ & - & 0.1 & $0.2,0.01$ & 2 & $14 \%$ & 1.0 \\
\hline
\end{tabular}




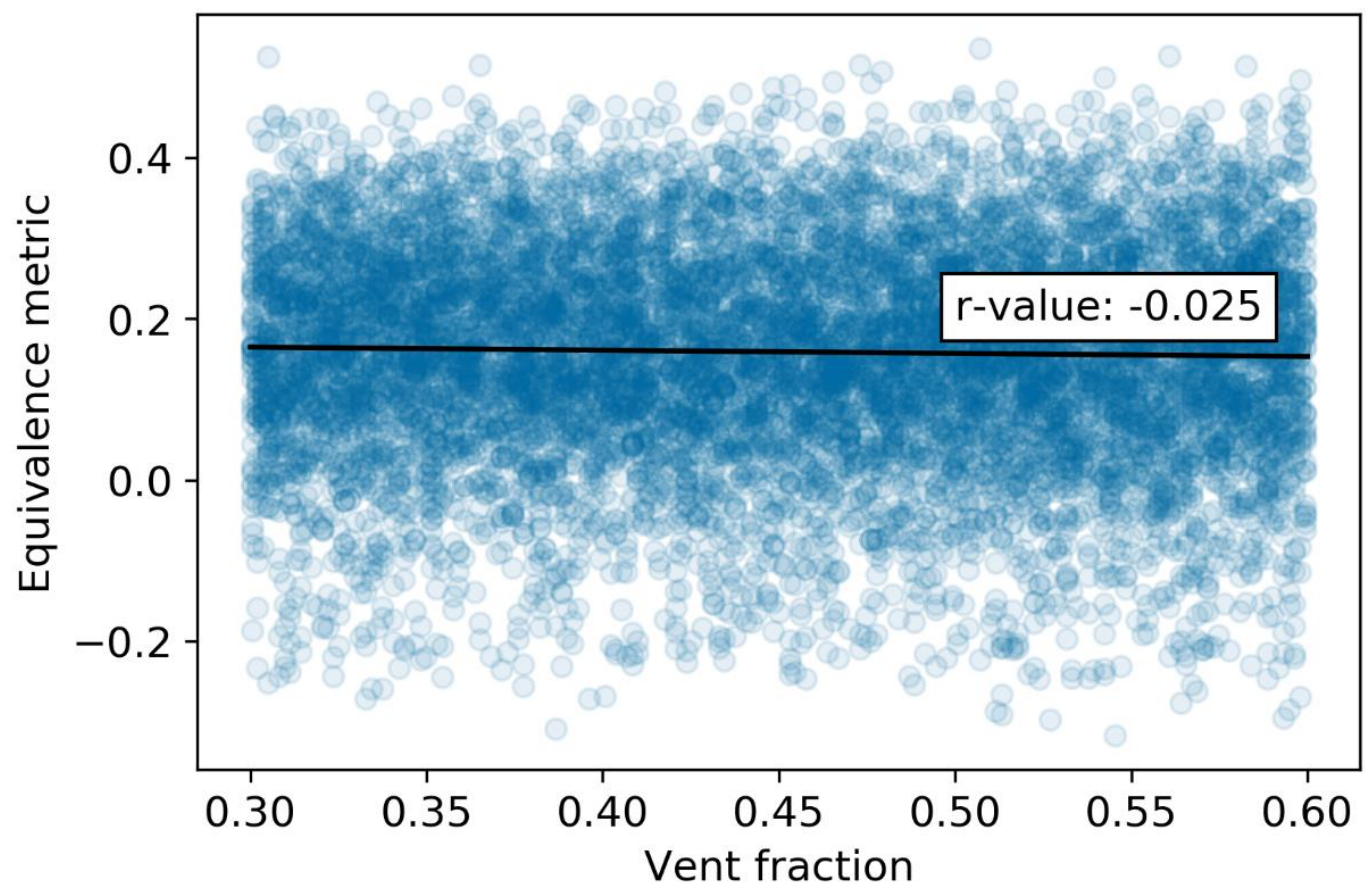

568

Figure S7 Equivalence metric of all iterations plotted against the vent fraction under UDIM. The best linear fit to the data is plotted along with the correlation coefficient of the fit.

In order to assess the value of reducing uncertainty in these four global parameters, we adopt a first order sensitivity index $S_{i}$ [24]. $S_{i}$ is defined by Equation (S8). $V_{X_{i}}$ is a variance operator with one parameter $(i)$ held constant. $E_{X_{\sim i}}$ is an expectation value operator across all variables except $i . \phi$ is the equivalence ratio defined in Equation (S7), and $X$ is the set of input parameters varied for the sensitivity analysis.

$$
S_{i}=\frac{V_{X_{i}}\left(E_{X_{\sim i}}\left(\phi \mid X_{i}\right)\right)}{V(\phi)}
$$

576 Figure S8 shows $S_{i}$ for the vent fraction, UDIM repair rate, leak production rate and emission 577 distribution. Eliminating uncertainty in the parameter with the largest $S_{i}$ would cause the greatest 578 decrease in variance of the equivalence ratio $V(\phi)$. Therefore, reducing uncertainty in the leak 579 production rate will have the greatest impact on uncertainty in equivalence analyses. 


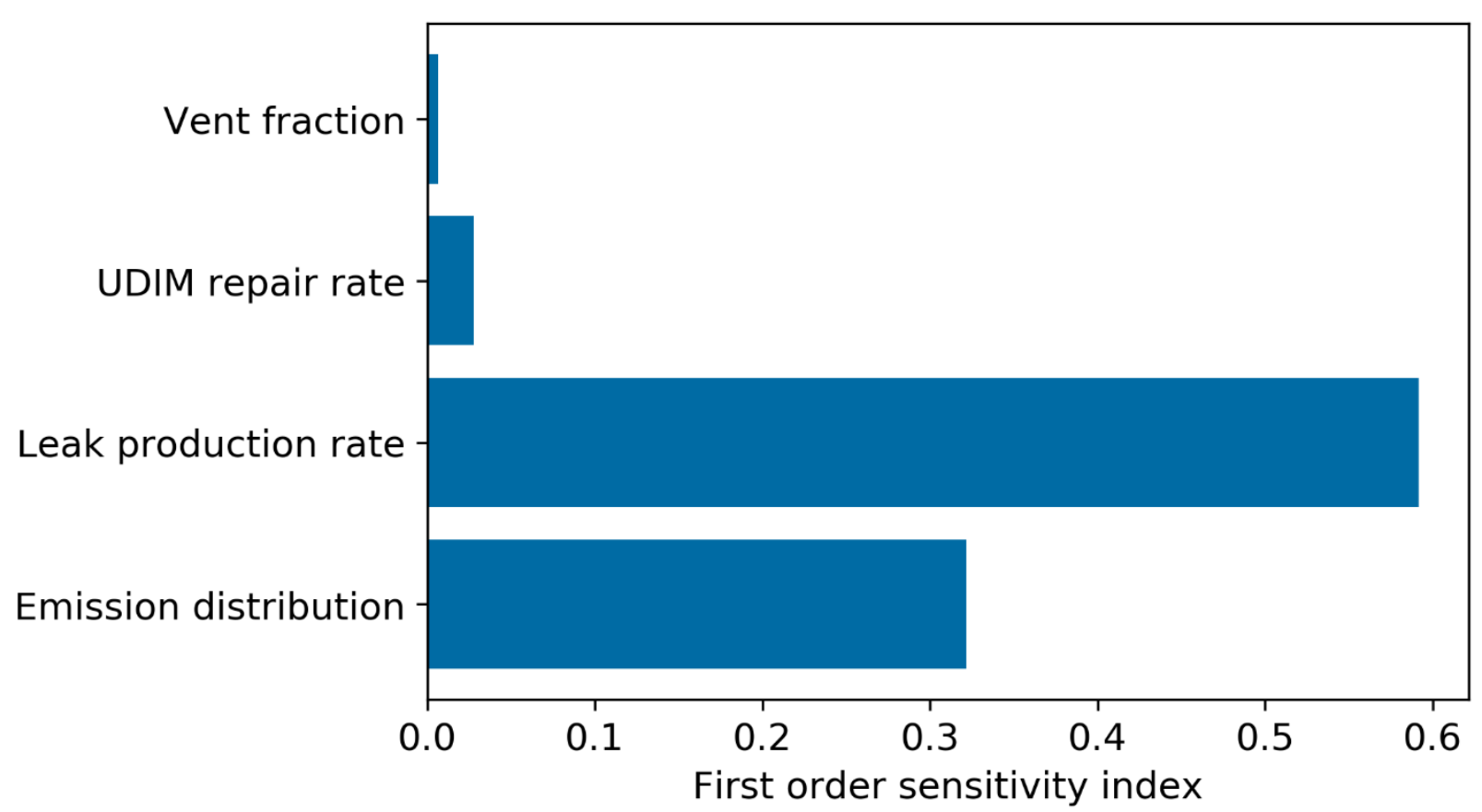

580

Figure S8 Sensitivity index of the four parameters tested in the sensitivity analysis.

\section{S4.7 Emissions variability versus detection variability}

The uncertainty in FEAST emission estimates is distinct from the uncertainty in equivalency estimates. Figure S9 shows the average emission rate in 100 iterations of FEAST with the same emission scenario. A UDIM scenario, a biannual OGI survey and a biannual Plane + OGI survey are considered. The emission rates shown are averaged across the 3-year simulation period and 100 well pads simulated in each iteration. In the UDIM scenario, the average fugitive emission rate ranges from 7 to $19 \mathrm{~kg} / \mathrm{day}$-site. In the Plane + OGI scenario, the rate ranges from 5 to 11 $\mathrm{kg} /$ day-site and in the OGI scenario the rate ranges from 3 to $7 \mathrm{~kg} /$ day-site. The ranges of the average site-level emissions overlap across the three scenarios. However, the ranking of the programs is consistent across each individual iteration - i.e., the OGI survey results in the least fugitive emissions, followed by the Plane + OGI and then the UDIM scenario. Figure S9 shows uncertainty in total emission estimates does not necessarily cause proportionate uncertainty in comparisons between LDAR programs. That is, the relative performance of different programs is significantly less uncertain than the range of fugitive emissions observed. 


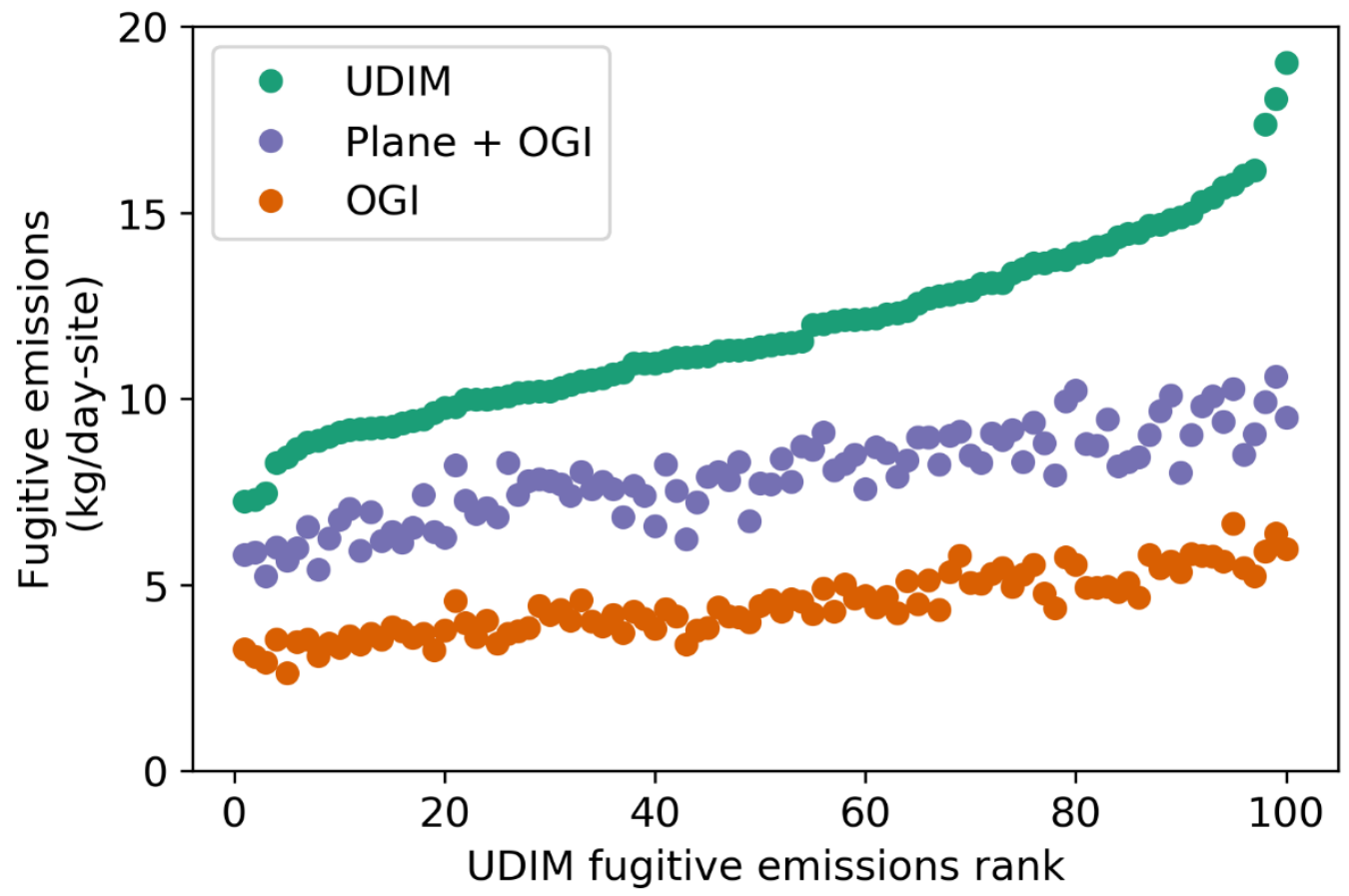

601

602

603

604

605

606
Figure S9 Average site-level fugitive emission rate under distinct LDAR scenarios, sorted by the UDIM fugitive emission rate. Results represent average emissions across a 3-year simulation period over 100 well pads.

\section{S5. Supporting Information References}

[1] COGCC, "COGCC/Data Downloads/GIS/Well Surface Location Data (Updated Daily)/Well Spots (APIs)," Colorado Oil and Gas Conservation Commission, 2019. https://cogcc.state.co.us/documents/data/downloads/gis/WELLS_SHP.ZIP (accessed Jan. 13, 2020).

[2] M. Omara et al., "Methane Emissions from Natural Gas Production Sites in the United States: Data Synthesis and National Estimate," Environ. Sci. Technol, vol. 52, p. 51, 2018, doi: 10.1021/acs.est.8b03535.

[3] 'Oil and Natural Gas Sector : Standards for Crude Oil and Natural Gas Facilities: Background Technical Support Document for the Proposed New Source Performance Standards 40 CFR Part 60, subpart OOOOa.” EPA, Chapel Hill, NC, 2016, [Online]. Available: https://beta.regulations.gov/document/EPA-HQ-OAR-2010-0505-7631.

[4] A. P. Ravikumar et al., "Repeated leak detection and repair surveys reduce methane emissions over scale of years," Environ. Res. Lett., 2020, doi: 10.1088/1748-9326/ab6ae1.

[5] Control of Ozone via Ozone Precursors and Control of Hydrocarbons via Oil and Gas Emissions. 2019. 
Non - peer reviewed pre - print submitted to EarthArXiv

623 [6] D. Allen et al., "Measurements of methane emissions at natural gas production sites in the United States David," Proc. Natl. Acad. Sci., vol. 110, no. 44, pp. 18025-18030, Sep. 2013, doi: 10.1073/pnas.1315099110.

[7] "City of Fort Worth Natural Gas Air Quality Study," Fort Worth, TX, 2011. [Online]. Available: http://fortworthtexas.gov/uploadedFiles/Gas_Wells/AirQualityStudy_final.pdf.

[8] J. Kuo, "Final project report estimation of methane emissions from the California Energy Commission," 2012. doi: 500-09-007.

[9] C. S. Bell et al., "Comparison of methane emission estimates from multiple measurement techniques at natural gas production pads," Elem Sci Anth, vol. 5, no. 0, p. 79, Dec. 2017 , doi: 10.1525/elementa.266.

[10] U.S. Environmental Protection Agency, "Inventory of U.S. Greenhouse Gas Emissions and Sinks: 1990-2018 - EPA 430-R-20-002,” Apr. 2020. Accessed: Aug. 13, 2020. [Online]. Available: https://www.epa.gov/ghgemissions/inventory-us-greenhouse-gasemissions-and-sinks.

[11] D. T. Allen et al., "Methane Emissions from Process Equipment at Natural Gas Production Sites in the United States: Pneumatic Controllers," 2014, doi: 10.1021/es5040156.

[12] A. R. Brandt, G. A. Heath, and D. Cooley, "Methane Leaks from Natural Gas Systems Follow Extreme Distributions," 2016, doi: 10.1021/acs.est.6b04303.

[13] U.S. Environmental Protection Agency, "Facility Level Information on Greenhouse Gases Tool,” 2017. https://ghgdata.epa.gov/ghgp/main.do\#/listFacilityForBasin/?q=Find a Facility or Location $\& b s=540 \&$ fid $=\& s f=11001000 \& l o w E=-$ 20000\&highE=23000000\&g1=1\&g2=1\&g3=1\&g4=1\&g5=1\&g6=0\&g7=1\&g8=1\&g9=1 $\& \mathrm{~g} 10=1 \& \mathrm{~g} 11=1 \& \mathrm{~g} 12=1 \& \mathrm{~s} 1=0 \& \mathrm{~s} 2=0 \& \mathrm{~s} 3=0 \& \mathrm{~s} 4=0 \& \mathrm{~s} 5=0 \& \mathrm{~s} 6=0 \& \mathrm{~s} 7=0 \& \mathrm{~s} 8=0 \& \mathrm{~s} 9=1 \& \mathrm{~s} 1$ $0=0 \&$ (accessed Oct. 23, 2019).

[14] G. G. Zaimes et al., "Characterizing Regional Methane Emissions from Natural Gas Liquid Unloading," 2019, doi: 10.1021/acs.est.8b05546.

[15] Mandatory Greenhouse Gas Reporting Subpart W--Petroleum and Natural Gas Systems. US, 2020.

[16] Mandatory Greenhouse Gas Reporting Subpart W--Petroleum and Natural Gas Systems: Calculating GHG Emisions. 2020.

[17] C. E. Kemp, A. P. Ravikumar, and A. R. Brandt, "Comparing Natural Gas Leakage Detection Technologies Using an Open-Source 'virtual Gas Field' Simulator," Environ. Sci. Technol., vol. 50, no. 8, 2016, doi: 10.1021/acs.est.5b06068.

[18] A. P. Ravikumar, J. Wang, M. Mcguire, C. S. Bell, D. Zimmerle, and A. R. Brandt, "Good versus Good Enough? Empirical Tests of Methane Leak Detection Sensitivity of a Commercial Infrared Camera," Environ. Sci. Technol, vol. 52, 2018, doi: 10.1021/acs.est.7b04945.

[19] T. E. Barchyn, C. H. Hugenholtz, and T. A. Fox, "Plume detection modeling of a dronebased natural gas leak detection system," Elem Sci Anth, vol. 7, no. 1, p. 41, Oct. 2019, doi: 10.1525/elementa.379.

[20] D. Zimmerle, T. Vaughn, C. Bell, K. Bennett, P. Deshmukh, and E. Thoma, "Detection Limits of Optical Gas Imaging for Natural Gas Leak Detection in Realistic Controlled Conditions," Environ. Sci. Technol., vol. 54, no. 18, pp. 11506-11514, 2020, doi: 10.1021/acs.est.0c01285.

[21] T. A. Fox, T. E. Barchyn, D. Risk, A. P. Ravikumar, and C. H. Hugenholtz, "A review of 
close-range and screening technologies for mitigating fugitive methane emissions in upstream oil and gas," Environ. Res. Lett., vol. 14, no. 5, p. 053002, Apr. 2019, doi: 10.1088/1748-9326/ab0cc3.

[22] A. Ravikumar et al., "Single-blind Inter-comparison of Methane Detection Technologies-Results from the Stanford/EDF Mobile Monitoring Challenge,” 2019, [Online]. Available: https://doi.org/10.1525/elementa.373.

[23] G. Myhre et al., "Chapter 8: Anthropogenic and Natural Radiative Forcing," in Climate Change 2013: The Physical Science Basis. Contribution of Working Group I to the Fifth Assessment Report of the Intergovernmental Panel on Climate Change, T. F. Stocker, D. Qin, G.-K. Plattner, M. Tignor, S. K. Allen, J. Boschung, A. Nauels, Y. Xia, V. Bex, and P. M. Midgley, Eds. Cambridge, United Kingdom and New York, NY, USA, 2013, pp. 659-740.

[24] A. Saltelli and P. Annoni, "How to avoid a perfunctory sensitivity analysis," Environ. Model. Softw., vol. 25, pp. 1508-1517, 2010, doi: 10.1016/j.envsoft.2010.04.012.

[25] C. E. Kemp, A. P. Ravikumar, and A. R. Brandt, "Comparing Natural Gas Leakage Detection Technologies Using an Open-Source Virtual Gas Field Simulator," Environ. Sci. Technol, 2016, doi: 10.1021/acs.est.5b06068.

[26] T. A. Fox, M. Gao, T. E. Barchyn, Y. L. Jamin, and H. Chris, "An agent-based model for estimating emissions reduction equivalence among leak detection and repair programs," pp. 1-44.

[27] M. R. Johnson, D. R. Tyner, S. Conley, S. Schwietzke, and D. Zavala-Araiza, "Comparisons of Airborne Measurements and Inventory Estimates of Methane Emissions in the Alberta Upstream Oil and Gas Sector," 2017, doi: 10.1021/acs.est.7b03525.

[28] D. R. Johnson, A. N. Covington, and N. N. Clark, "Methane Emissions from Leak and Loss Audits of Natural Gas Compressor Stations and Storage Facilities," Environ. Sci. Technol, vol. 49, p. 35, 2015, doi: 10.1021/es506163m.

[29] D. Zimmerle et al., "Methane Emissions from Gathering Compressor Stations in the U.S.," Environ. Sci. Technol., vol. 54, no. 12, pp. 7552-7561, Jun. 2020, doi: 10.1021/acs.est.0c00516. 